UNITED STATES DEPARTMENT OF THE INTERIOR

GEOLOGICAL SURVEY

\title{
Analytical results for total and partial metal extractions in aquifer material, Plnal Creek, Globe, Arizona
}

By

Walter H. Ficklin, ${ }^{1}$ Alonza H. Love, ${ }^{1}$ and Paul K. Briggs ${ }^{1}$

Open-File Report 91-111

This report is prelininary and has not been reviewed for conformity with U.S. Geological Survey editorial standards and stratigraphic nomenclature. Any use of trade names is for descriptive purposes only and does not imply endorsement by the USGS.

${ }^{1}$ U.s. Geological Survey, DFC, Box 25046, MS 973, Denver, CO 80225 


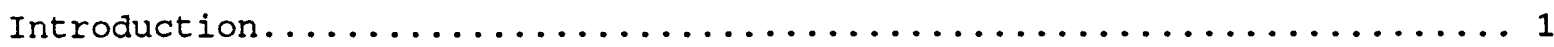

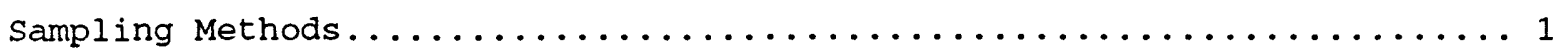

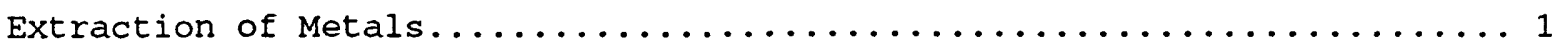

References......................................

\section{Illustrations}

Figure 1. Map showing sample collection sites near Globe, Arizona..... 3

Figure 2. Generalized cross section of Pinal Creek aquifer near

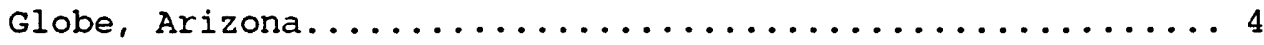

\section{Tables}

Table 1 sequential extraction scheme........................ 5

Table 2 Analytical results for total metal concentration of -80 fraction of samples from holes in the Pinal Creek aquifer, Globe, Arizona........................... 6

Table 3 Analytical results for partial metal extractions,

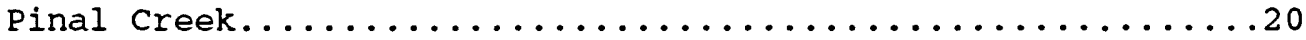




\section{Analytical Results for Total and Partial Metal Extractions in Aquifer Material, Pinal Creek, Globe, Arizona}

By

Walter H. Ficklin, Alonza H. Love, and Paul K. Briggs

\section{INTRODUCTION}

Acidic water has been found in samples collected from the Pinal creek aquifer near Globe, Arizona (Eychaner, 1987; Eychaner and Stollenwerk, 1987; and Eychaner, 1988). As the water reacts with the aquifer material some of the acidity is neutralized. Eventually the entire aquifer will become acidic at which time the acid water will emerge at Pinal creek, a permanent stream that flows northward from the end of the aquifer. In order to assess the effect of the water-rock interaction, samples were collected during well construction at sites X1, 451, and X5 (figs. 1 and 2). A background hole was drilled at site 010 (fig. 1). Hole 452 is located at site 451 and holes 505 and 506 are located at site $\mathrm{X} 5$.

\section{SAMPLING METHODS}

Each hole was drilled using a split-spoon auger. Samples were collected at random intervals down the hole to represent changes in the aquifer. Samples were collected at about 10-ft intervals in each hole to about 75-80 ft. In some holes, samples were collected at much closer spacing.

\section{EXTRACTION OF METALS}

Each sample was sieved through an 80 -mesh sieve. The -80 portion of each sample was used in the total metal determination (Lichte and others, 1987) as well as the partial extractions. We extracted each sample according to the scheme described in table 1 . These extraction techniques are described in detail by chao (1984). Results for total-metal analysis are presented in table 2. Results for partial-sequential analysis are presented in table 3 . All metal determinations for the partial extractions were made using conventional atomic-à jorption spectrophotometry.

The series of partial-sequential extractions described in table 1 are usually done with the analysis of metals as the objective. However, the acid water contains large concentrations of sulfate, which may react with the aquifer material to produce insoluble sulfate compounds. Sulfate was determined from the manganese oxide and amorphous iron-oxide solutions following matrix modification with Dionex Ag cleanup columns. The cleanup columns remove chloride from solution, and the solutions contain sufficient chloride to prevent direct determination by ion chromatography (IC). Following cleanup, sulfate determination was done by conventional IC. Sulfate results are presented in table 3. Total sulfur, expressed as sulfate, was determined using a Leco Sc132 sulfur determinator. The carbonate solutoins were diluted one hudred fold prior to direct injection in the IC. 


\section{REFERENCES}

Chao, T.T., 1984, Use of partial dissolution techniques in geochemical exploration, Journal of Geochemical Exploration, 20, p. 101-135.

Eychaner, J.H., 1988, Movement of inorganic contaminants in acidic water near Globe, Arizona: Proceedings of the Technical Meeting, Phoenix, Arizona, Sept 26-30, 1988, U.S. Geological survey Toxic substances Hydrology Program, Gail E. Mallard and stephen E. Ragone, eds., U.S. Geological Survey Water Resources Investigation Report 88-4220, p. 567.

Eychaner, J.H., and stollenwerk, K.G., 1987, Acid groundwater contamination from copper mining near Globe, Arizona I, Proceedings of the Third Technical Meeting, U.S. Geological survey program on toxic waste: Groundwater contamination, Pensacola, Florida, March 23-27, 1987, Bernard J. Franks, ed., U.S. Geological Survey open-File Report 87-109, p. D13.

Lichte, F.E., Golightly, D.W., and LaMothe, P.J., 1987, Inductively coupled plasma atomic emission spectrometry, in Baedecker, P.A., ed., U.S. Geological Survey Bulletin 1770, p. B1-B10.

Stollenwerk, K.G., and Eychaner, J.H.,1987, Acidic groundwater contamination from copper mining near Globe, Arizona: II, Neutralization capacity of alluvium, in Proceedings of the Third Technical Meeting, U.S. Geological Survey program on toxic waste: Groundwater contamination, Pensacola, Florida, March 23-27, 1987, Bernard J. Franks, ed., U.S. Geological Survey Open-File Report 87-109, p. D19.

Stollenwerk, K.G., and Eychaner, J.H., 1988, Solubility of aluminum and iron in ground water near Globe, Arizona: Proceedings of the Technical Meeting, Phoenix, Arizona, sept 26-30, 1988, U. S. Geological Survey Toxic Substances Hydrology Program, Gail E. Mallard and stephen E. Ragone, eds., U.S. Geological Survey Water Resources Investigation Report 88-4220, p. 567. 


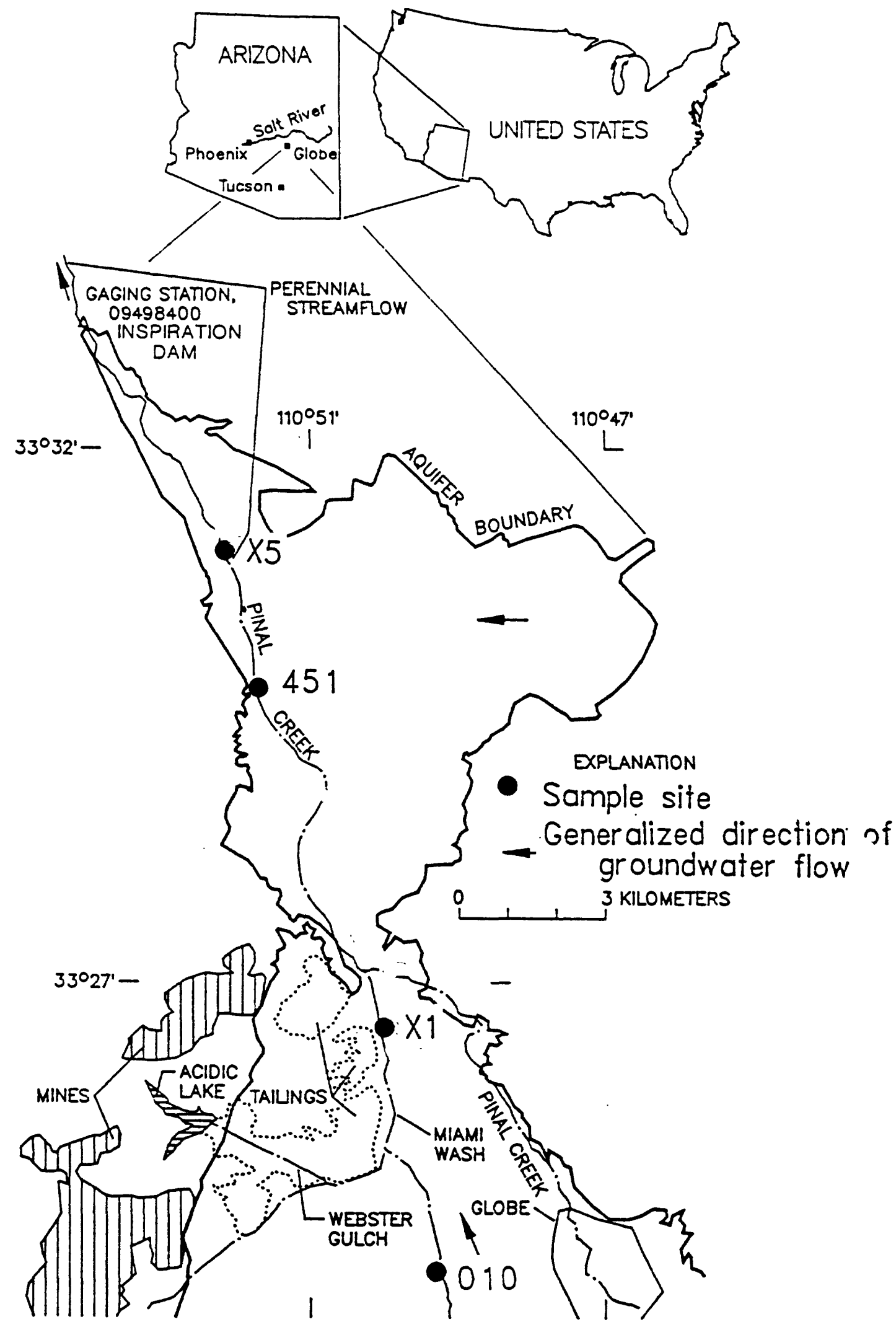

Figure 1. Sample collection sites near Globe, Arizona. 


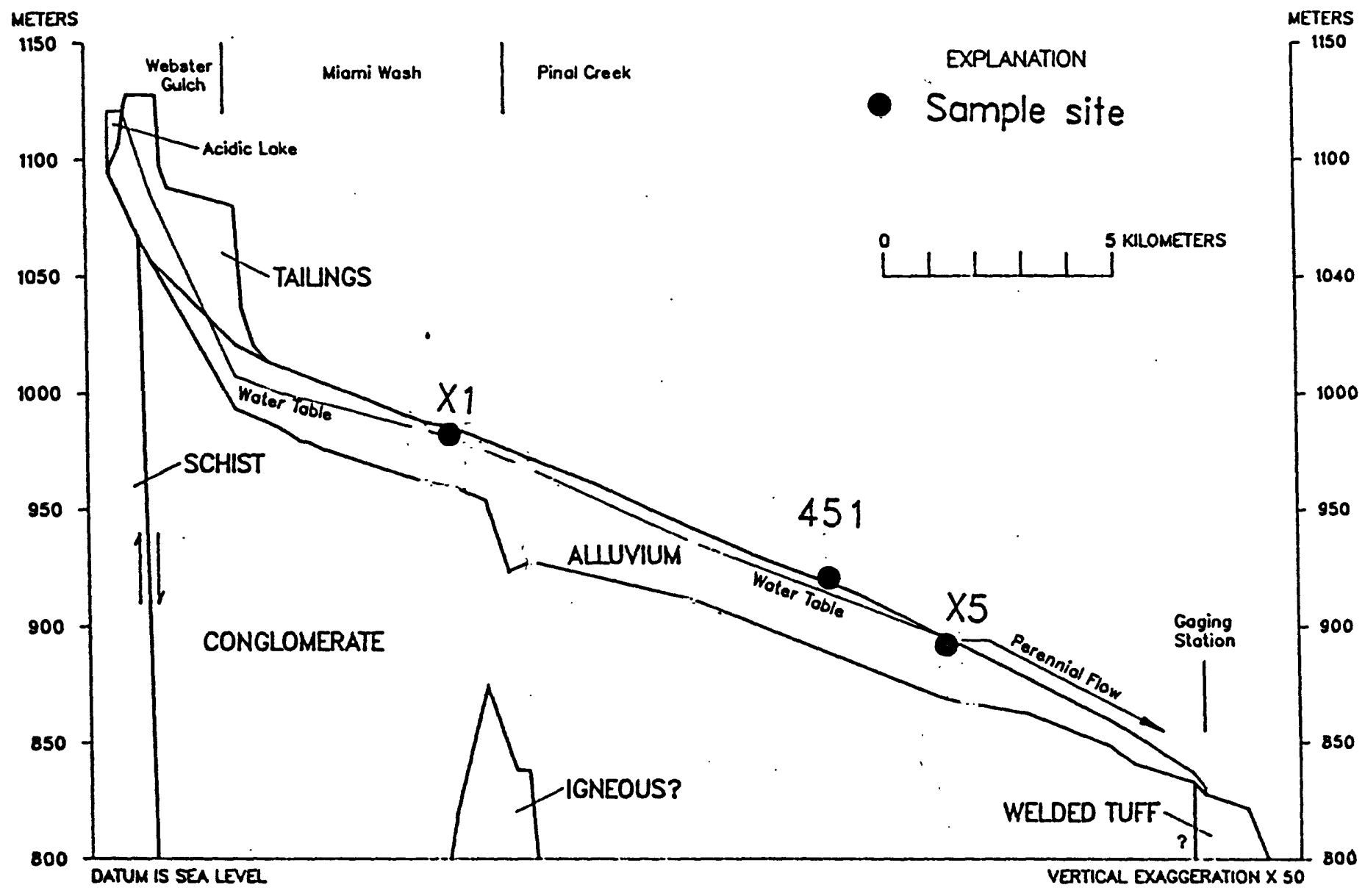

Figure 2. Generalized cross section of Pinal Creek aquifer near Globe, Arizona. 
Table 1. Sequential extraction scheme

[g, gram; mL, milliliter; ${ }^{\circ} \mathrm{C}$, degrees Centigrade]

Step 1. Dissolution of carbonate and related elements

Step 2. Dissolution of manganese oxides and related elements

Step 3. Dissolution of amorphous iron oxides and related elements

Step 4. Dissolution of crystalline iron oxide and related elements

Step 5. Dissolution of residual material
$1 \mathrm{~g}$ of sample plus $20 \mathrm{~mL}$ of 1. $\mathrm{OM}$ acetic acid-sodium acetate buffer pH 5--shake for $4 \mathrm{hrs}$

residue from step 1 plus $25 \mathrm{~mL}$ of $0.1 \mathrm{~N}$ hydroxylamine hydrochloride in $0.01 \mathrm{~N}$ nitric acid--shake for $30 \mathrm{~min}$

residue from step 2 plus $25 \mathrm{~mL}$ of $0.25 \mathrm{~N}$ hydroxylamine hydrochloride in $0.25 \mathrm{~N}$ hydrochloric acid--heat in $70{ }^{\circ} \mathrm{C}$ water bath for $30 \mathrm{~min}$

residue from step 3 plus $25 \mathrm{~mL}$ of $4.0 \mathrm{~N}$ and related elements hydrochloric acid--shake in $90^{\circ} \mathrm{C}$ water bath for $30 \mathrm{~min}$

residue from step 4 is digested in hydrofluoric, perchloric and nitric acid and taken up in $25 \mathrm{~mL}$ of $0.5 \mathrm{~N}$ nitric acid 
Table 2. Analytical results for total metal concentration of -80 fraction of samples from holes in the Pinal Creek aquifer, Globe, Arizona

[\%, percent; $\mathrm{mg} / \mathrm{kg}, \mathrm{milligrams}$ per killogram]

\begin{tabular}{|c|c|c|c|c|c|c|c|}
\hline Sample & & th & $\begin{array}{l}\text { Al } \\
8\end{array}$ & $\begin{array}{l}\mathrm{Ca} \\
q\end{array}$ & $\begin{array}{l}\mathrm{Fe} \\
\text { \& }\end{array}$ & $\begin{array}{l}K \\
8\end{array}$ & $\begin{array}{l}\mathrm{Mg} \\
\&\end{array}$ \\
\hline $\mathrm{x} 1-107 \mathrm{bl}$ & 16 & & 7.45 & 2.62 & 8.37 & 1.79 & 1.1 \\
\hline$x_{1}-107 b 2$ & 17 & & 8.47 & 2.02 & 5.12 & 1.71 & 1.29 \\
\hline$x 1-107 b 3$ & 17. & & 7.94 & 1.78 & 4.77 & 2.29 & 0.98 \\
\hline$x 1-107 b 4$ & 18 & & 8.00 & 1.79 & 5.19 & 2.5 & 0.83 \\
\hline$x 1-107 b 5$ & 19 & -24 & 7.54 & 1.98 & 6.54 & 2.04 & 0.83 \\
\hline$x 1-107 b 6$ & 38 & -39 & 8.01 & 1.92 & 6.27 & 2.25 & 1.05 \\
\hline$x \times 1-107 b 7$ & 46 & -47 & 7.36 & 1.87 & 9.64 & 2.06 & 0.87 \\
\hline$x 1-107 b 8$ & 56 & -57 & 7.39 & 1.90 & 9.42 & 2.17 & 0.92 \\
\hline$x 1-107 b 9$ & 72 & -72 & 7.25 & 2.05 & 10.7 & 2.04 & 0.92 \\
\hline$x 5-505 b 1$ & & $7-1.1$ & 5.83 & 2.34 & 11.0 & 1.82 & 1.17 \\
\hline$x 5-505 b 2$ & & $1-2$ & 5.20 & 1.83 & 18.0 & 1.6 & 0.92 \\
\hline$x 5-505 b 3$ & 9 & -10 & 7.20 & 2.51 & 8.26 & 2.12 & 1.42 \\
\hline$x 5-505 b 4$ & 10 & -11.5 & 7.81 & 2.32 & 5.83 & 2.16 & 1.41 \\
\hline$x 5-505 b 5$ & 18 & -21.5 & 6.46 & 2.52 & 11.8 & 1.8 & 1.31 \\
\hline$x 5-505 b 6$ & 23 & -24.8 & 6.71 & 2.66 & 10.2 & 1.73 & 1.44 \\
\hline$x 5-505 b 7$ & 58 & -60 & 7.40 & 2.26 & 7.69 & 2.09 & 1.33 \\
\hline$x 5-505 b 8$ & 60 & -61.5 & 7.16 & 2.40 & 9.16 & 2.17 & 1.34 \\
\hline$x 5-505 b 9$ & 64 & -65 & 7.34 & 3.37 & 4.75 & 1.64 & 1.34 \\
\hline$x 5-505 b 10$ & 71 & -73 & 7.04 & 4.55 & 3.03 & 1.82 & 1.03 \\
\hline$x 5-506 b 1$ & 0. & $3-\quad 1.2$ & 5.52 & 2.04 & 13.2 & 1.89 & 1.04 \\
\hline$x 5-506 b 2$ & 10. & $5-11.5$ & 8.06 & 2.43 & 6.77 & 2.10 & 1.61 \\
\hline$x 5-506 b 3$ & 19 & -22 & 7.83 & 2.3 & 6.32 & 2.53 & 1.40 \\
\hline$x 5-506$ & 23 & -24.8 & 7.15 & 2.66 & 8.19 & 1.88 & 1.56 \\
\hline $451 \mathrm{bl}$ & 10. & & 7.12 & 2.57 & 10.7 & 2.7 & 1.48 \\
\hline $451 \mathrm{~b} 2$ & 12 & & 7.18 & 2.54 & 9.5 & 2.76 & 1.41 \\
\hline $451 \mathrm{b3}$ & 12 & & 7.52 & 2.60 & 9.49 & 2.84 & 1.54 \\
\hline $451 \mathrm{~b} 4$ & 13 & . & 7.22 & 1.97 & 7.15 & 1.95 & 0.74 \\
\hline 451 & 21 & & 7.41 & 4.00 & 6.80 & 2.23 & 2.51 \\
\hline $451 b 5$ & 30 & -31 & 6.9 & 2.56 & 11.7 & 1.98 & 1.46 \\
\hline $451 \mathrm{~b} 6$ & 32 & & 7.59 & 2.59 & 8.45 & 2.07 & 1.57 \\
\hline $451 b 7$ & 36 & & 6.41 & 2.56 & 13.1 & 1.49 & 1.55 \\
\hline $451 b 8$ & 37. & $5-38$ & 5.62 & 2.1 & 20.7 & 1.41 & 2.43 \\
\hline $451 b 9$ & 42. & & 7.63 & 2.55 & 9.83 & 2.26 & 1.67 \\
\hline 451 & 51. & $8-52.3$ & 7.40 & 2.38 & 6.57 & 2.51 & 1.47 \\
\hline $451 b 10$ & 57 & & 5.21 & 2.21 & 21.2 & 1.36 & 1.33 \\
\hline $451 b 11$ & 70 & -73 & 5.94 & 2.35 & 16.1 & 1.58 & 1.36 \\
\hline $451 b 12$ & 76. & $5-70$ & 7.15 & 1.98 & 11.1 & 1.98 & 1.24 \\
\hline $452 \mathrm{bl}$ & 3 & -6 & 6.43 & 2.73 & 13.5 & 1.66 & 1.45 \\
\hline $452 \mathrm{~b} 2$ & & $5-7$ & 4.24 & 2.18 & 26.2 & 1.00 & 1.13 \\
\hline
\end{tabular}


Table 2. Analytical results for total metal concentration of -80 fraction of samples from holes in the Pinal Creek aquifer, Globe, Arizona--(Continued)

\begin{tabular}{|c|c|c|c|c|c|c|c|}
\hline Sample & $\begin{array}{l}\text { Dep } \\
\text { (fe }\end{array}$ & th & $\begin{array}{l}\text { Al } \\
\text { \& }\end{array}$ & $\begin{array}{l}\mathrm{Ca} \\
8\end{array}$ & $\begin{array}{l}\mathrm{Fe} \\
8\end{array}$ & $\begin{array}{l}K \\
8\end{array}$ & $\begin{array}{l}\mathrm{Mg} \\
\mathrm{q}\end{array}$ \\
\hline $452 \mathrm{~b} 3$ & 11 & -12 & 7.99 & 2.18 & 5.52 & 2.28 & 1.52 \\
\hline 452 & 14.8 & $3-15$ & 8.85 & 1.62 & 4.60 & 2.17 & 1.51 \\
\hline $452 \mathrm{~b} 4$ & 18 & -19 & 7.39 & 2.53 & 9.00 & 2.05 & 1.45 \\
\hline $452 b 5$ & 19 & -21 & 6.33 & 2.78 & 14.2 & 1.61 & 1.55 \\
\hline $452 \mathrm{~b} 6$ & 23 & -23 & 6.38 & 2.33 & 16.2 & 1.62 & 1.34 \\
\hline 010b1 & 5 & -10 & 7.61 & 3.22 & 10.9 & 1.50 & 1.38 \\
\hline $010 b 2$ & 15 & -20 & 7.02 & 2.40 & 9.96 & 1.62 & 1.05 \\
\hline 010b3 & 23 & -25 & 8.10 & 1.88 & 6.45 & 2.12 & 1.14 \\
\hline $010 b 4$ & 29 & -30 & 7.48 & 2.77 & 10.6 & 1.47 & 1.29 \\
\hline 010b5 & 37 & -38 & 7.91 & 2.22 & 7.58 & 1.83 & 1.25 \\
\hline $010 b 6$ & 45 & -46 & 7.76 & 1.97 & 7.93 & 2.08 & 1.1 \\
\hline 010b7 & 50 & -52 & 7.84 & 1.5 & 5.91 & 2.09 & 1.01 \\
\hline $010 b 8$ & 57.5 & -60 & 7.64 & 1.83 & 5.66 & 1.88 & 1.18 \\
\hline 010b9 & 60 & -63 & 6.1 & 1.35 & 3.58 & 1.75 & 0.78 \\
\hline $010 b 10$ & 63 & -67 & 7.82 & 2.47 & 4.27 & 2.03 & 1.37 \\
\hline 010b11 & 70 & -75 & 7.66 & 6.11 & 3.97 & 1.65 & 1.45 \\
\hline $010 \mathrm{~b} 12$ & 86 & -90 & 8.13 & 4.05 & 4.89 & 1.60 & 1.56 \\
\hline
\end{tabular}


Table 2. Analytical results for total metal concentration of -80 fraction of samples from holes in the Pinal Creek aquifer, Globe, Arizona--(Continued)

\begin{tabular}{|c|c|c|c|c|c|c|c|c|}
\hline Sample & & $\begin{array}{l}\text { pth } \\
\text { eet }\end{array}$ & & $\begin{array}{l}\mathrm{Na} \\
8\end{array}$ & $\begin{array}{l}P \\
8\end{array}$ & $\begin{array}{l}\mathrm{Ti} \\
8\end{array}$ & $\begin{array}{c}\mathrm{Mn} \\
\mathrm{mg} / \mathrm{kg}\end{array}$ & $\begin{array}{l}\mathrm{As} \\
\mathrm{mg} / \mathrm{kg}\end{array}$ \\
\hline$x 1-107 b 1$ & 16 & & & 1.58 & 0.14 & 1.00 & 1,400 & $<10$ \\
\hline$x 1-107 b 2$ & 17 & & & 1.46 & 0.13 & 0.53 & 1,200 & $<10$ \\
\hline$x 1-107 b 3$ & 17. & & & 1.61 & 0.13 & 0.49 & 23,800 & $<10$ \\
\hline$x 1-107 b 4$ & 18 & & & 1.93 & 0.11 & 0.49 & 729 & $<10$ \\
\hline$x 1-107 b 5$ & 19 & - & 24 & 1.87 & 0.11 & 0.74 & 908 & $<10$ \\
\hline$x 1-107 b 6$ & 38 & - & 39 & 1.72 & 0.12 & 0.63 & 850 & 10 \\
\hline$x 1-107 b 7$ & 46 & - & 47 & 1.61 & 0.13 & 0.92 & 1,070 & $<10$ \\
\hline$x 1-107 b 8$ & 56 & - & 57 & 1.64 & 0.12 & 0.98 & 1,060 & $<10$ \\
\hline x1-107b9 & 71 & - & 72 & 1.48 & 0.13 & 0.91 & 990 & $<10$ \\
\hline$x 5-505 b 1$ & 0 . & $7-$ & 1.1 & 1.27 & 0.13 & 2.20 & 44,600 & $<10$ \\
\hline$x 5-505 b 2$ & 1. & $1-$ & 2 & 1.24 & 0.14 & 3.16 & 3,070 & $<10$ \\
\hline$x 5-505 b 3$ & 9 & - & 10 & 1.75 & 0.15 & 1.65 & 2,000 & 10 \\
\hline$x 5-505 b 4$ & 10 & - & 11.5 & 1.67 & 0.14 & 0.93 & 5,320 & 10 \\
\hline$x 5-505 b 5$ & 18 & - & 21.5 & 1.62 & 0.16 & 2.52 & 2,080 & $<10$ \\
\hline$x 5-505 b 6$ & 23 & - & 24.8 & 1.49 & 0.24 & 2.42 & 2,010 & 10 \\
\hline$x 5-505 b 7$ & 58 & - & 60 & 1.54 & 0.16 & 1.33 & 2,410 & 10 \\
\hline$x 5-505 b 8$ & 60 & - & 61.5 & 1.62 & 0.14 & 1.66 & 2,340 & 10 \\
\hline$x 5-505 b 9$ & 64 & - & 65 & 1.40 & 0.09 & 0.85 & 1,000 & 20 \\
\hline$x 5-505 b 10$ & 71 & - & 73 & 1.58 & 0.09 & 0.48 & 691 & 10 \\
\hline$x 5-506 b 1$ & 0. & $8-$ & 1.2 & 1.07 & 0.14 & 2.43 & 30,900 & 10 \\
\hline$x 5-506 b 2$ & 10. & $5-$ & 11.5 & 1.57 & 0.15 & 1.12 & 1,170 & 10 \\
\hline$x 5-506 b 3$ & 19 & - & 22 & 1.78 & 0.12 & 1.11 & 1,590 & $<10$ \\
\hline$x 5-506$ & 23 & - & 24.8 & 1.57 & 0.20 & 1.87 & 178 & 10 \\
\hline $451 b 1$ & 10. & & & 1.56 & 0.18 & 2.12 & 3,510 & $<10$ \\
\hline $451 b 2$ & 11 & & & 1.64 & 0.16 & 1.87 & 2,270 & $<10$ \\
\hline $451 b 3$ & 12 & & & 1.49 & 0.19 & 1.73 & 2,480 & $<10$ \\
\hline $451 b 4$ & 13 & & & 1.8 & 0.13 & 0.81 & 1,380 & $<10$ \\
\hline 451 & 21 & & & 1.64 & 0.11 & 1.21 & 1,610 & 10 \\
\hline $451 b 5$ & 30 & - & 31 & 1.61 & 0.13 & 2.56 & 2,160 & $<10$ \\
\hline $451 b 6$ & 32 & & & 1.73 & 0.15 & 1.57 & 2,210 & $<10$ \\
\hline $451 b 7$ & 36 & & & 1.27 & 0.23 & 2.9 & 2,490 & 10 \\
\hline $451 b 8$ & 37. & $5-$ & 38 & 1.12 & 0.16 & 4.11 & 3,150 & $<10$ \\
\hline 451b9 & 42 . & & & 1.74 & 0.12 & 1.99 & 1,940 & 10 \\
\hline 451 & 51. & $8-$ & 52.3 & 1.78 & 0.13 & 1.15 & 1,400 & $<10$ \\
\hline $451 b 10$ & 57 & & & 1.1 & 0.17 & 4.12 & 3,240 & 10 \\
\hline $451 \mathrm{~b} 11$ & 70 & - & 73 & 1.23 & 0.15 & 3.14 & 2,420 & $<10$ \\
\hline $451 b 12$ & 76. & $5-$ & 70 & 1.40 & 0.14 & 1.47 & 1,390 & $<10$ \\
\hline $452 \mathrm{bl}$ & 3 & - & 6 & 1.49 & 0.19 & 2.28 & 2,050 & 10 \\
\hline $452 \mathrm{~b} 2$ & & $5-$ & 7 & 0.98 & 0.16 & 4.63 & 3,530 & $<10$ \\
\hline
\end{tabular}


Table 2. Analytical results for total metal concentration of -80 fraction of samples from holes in the Pinal Creek aquifer, Globe, Arizona--(Continued)

\begin{tabular}{|c|c|c|c|c|c|c|c|}
\hline Sample & $\begin{array}{l}\text { Dep } \\
\text { (fe }\end{array}$ & $\begin{array}{l}\text { th } \\
\text { et) }\end{array}$ & $\begin{array}{l}\mathrm{Na} \\
\mathrm{8}\end{array}$ & $\begin{array}{l}\text { P } \\
8\end{array}$ & $\begin{array}{l}\mathrm{Ti} \\
8\end{array}$ & $\begin{array}{c}\mathrm{Mn} \\
\mathrm{mg} / \mathrm{kg}\end{array}$ & $\begin{array}{l}\mathrm{As} \\
\mathrm{mg} / \mathrm{kg}\end{array}$ \\
\hline $452 \mathrm{~b} 3$ & 11 & -12 & 1.63 & 0.12 & 0.79 & 2,730 & $<10$ \\
\hline 452 & 14.8 & -15 & 1.23 & 0.08 & 0.47 & 1,220 & $<10$ \\
\hline $452 \mathrm{~b} 4$ & 18 & -19 & 1.71 & 0.16 & 1.76 & 2,110 & $<10$ \\
\hline $452 \mathrm{~b} 5$ & 19 & -21 & 1.42 & 0.21 & 3.07 & 2,750 & $<10$ \\
\hline $452 \mathrm{~b} 6$ & 23 & -23 & 1.32 & 0.16 & 2.88 & 2,740 & $<10$ \\
\hline 010b1 & 5 & -10 & 1.31 & 0.18 & 0.81 & 1,230 & $<10$ \\
\hline 010b2 & 15 & -20 & 1.36 & 0.13 & 0.68 & 1,040 & $<10$ \\
\hline $010 \mathrm{b3}$ & 23 & -25 & 1.39 & 0.11 & 0.46 & 815 & $<10$ \\
\hline $010 b 4$ & 29 & -30 & 1.29 & 0.15 & 0.77 & 1,080 & $<10$ \\
\hline 010b5 & 37 & -38 & 1.31 & 0.13 & 0.56 & 887 & $<10$ \\
\hline $010 b 6$ & 45 & -46 & 1.26 & 0.11 & 0.57 & 986 & $<10$ \\
\hline 010b7 & 50 & -52 & 1.33 & 0.08 & 0.38 & 771 & $<10$ \\
\hline $010 b 8$ & 57.5 & ;- 60 & 1.3 & 0.13 & 0.49 & 741 & $<10$ \\
\hline 010b9 & 60 & -63 & 1 & 0.05 & 0.31 & 473 & $<10$ \\
\hline 010b10 & 63 & -67 & 0.89 & 0.08 & 0.36 & 655 & $<10$ \\
\hline $010 \mathrm{~b} 11$ & 70 & -75 & 0.87 & 0.08 & 0.34 & 686 & $<10$ \\
\hline $010 b 12$ & 86 & -90 & 0.84 & 0.09 & 0.38 & 709 & $<10$ \\
\hline
\end{tabular}


Table 2. Analytical results for total metal concentration of -80 fraction of samples from holes in the Pinal Creek aquifer, Globe, Arizona--(Continued)

\begin{tabular}{|c|c|c|c|c|c|c|c|}
\hline Sample & \multicolumn{2}{|c|}{$\begin{array}{l}\text { Depth } \\
\text { (feet) }\end{array}$} & $\begin{array}{c}\mathrm{Ba} \\
\mathrm{mg} / \mathrm{kg}\end{array}$ & $\begin{array}{c}\mathrm{Be} \\
\mathrm{mg} / \mathrm{kg}\end{array}$ & $\begin{array}{c}\mathrm{Ce} \\
\mathrm{mg} / \mathrm{kg}\end{array}$ & $\begin{array}{c}\mathrm{Co} \\
\mathrm{mg} / \mathrm{kg}\end{array}$ & $\begin{array}{c}\mathrm{Cr} \\
\mathrm{mg} / \mathrm{kg}\end{array}$ \\
\hline$x 1-107 b 1$ & \multicolumn{2}{|l|}{16} & 552 & 2 & 126 & 27 & 149 \\
\hline$x 1-107 b 2$ & \multicolumn{2}{|l|}{17} & 580 & 3 & 79 & 26 & 82 \\
\hline$x 1-107 b 3$ & \multicolumn{2}{|c|}{17.5} & 907 & 3 & 97 & 478 & 71 \\
\hline$x 1-107 b 4$ & \multicolumn{2}{|c|}{18} & 748 & 3 & 68 & 16 & 84 \\
\hline$x 1-107 b 5$ & 19 & -24 & 718 & 3 & 87 & 18 & 108 \\
\hline$x 1-107 b 6$ & 38 & -39 & 688 & 3 & 75 & 19 & 100 \\
\hline$x 1-107 b 7$ & 46 & -47 & 631 & 2 & 84 & 22 & 153 \\
\hline$x 1-107 b 8$ & 56 & -57 & 656 & 2 & 88 & 23 & 159 \\
\hline$x 1-107 b 9$ & 71 & -72 & 673 & 2 & 82 & 24 & 198 \\
\hline$x 5-505 b 1$ & & $7-1.1$ & 541 & 3 & 126 & 40 & 181 \\
\hline$x 5-505 b 2$ & & $1-2$ & 431 & 2 & 134 & 36 & 315 \\
\hline$x 5-505 b 3$ & 9 & -10 & 585 & 2 & 78 & 30 & 142 \\
\hline$x 5-505 b 4$ & 10 & -11.5 & 1,000 & 2 & 79 & 33 & 88 \\
\hline$x 5-505 b 5$ & 18 & -21.5 & 509 & 2 & 85 & 33 & 220 \\
\hline$x 5-505 b 6$ & 23 & -24.8 & 531 & 2 & 104 & 35 & 192 \\
\hline$x 5-505 b 7$ & 58 & -60 & 710 & 2 & 79 & 29 & 146 \\
\hline$x 5-505 b 8$ & 60 & -61.5 & 654 & 2 & 83 & 31 & 158 \\
\hline X5-505b9 & 64 & -65 & 753 & 2 & 63 & 19 & 63 \\
\hline$x 5-505 b 10$ & 71 & -73 & 647 & 2 & 56 & 14 & 39 \\
\hline$x 5-506 b 1$ & 0. & $8-\quad 1.2$ & 499 & 3 & 102 & 42 & 226 \\
\hline$x 5-506 b 2$ & 10. & $5-11.5$ & 570 & 2 & 89 & 27 & 91 \\
\hline$x 5-506 b 3$ & & -22 & 760 & 2 & 69 & 29 & 103 \\
\hline$x 5-506$ & & -24.8 & 560 & 2 & 89 & 31 & 148 \\
\hline $451 b 1$ & 10. & & 523 & 2 & 119 & 32 & 186 \\
\hline $451 b 2$ & 11 & & 519 & 2 & 90 & 31 & 160 \\
\hline $451 b 3$ & 12 & & 628 & 2 & 101 & 32 & 148 \\
\hline $451 b 4$ & 13 & & 674 & 3 & 98 & 18 & 124 \\
\hline 451 & 21 & 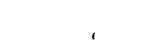 & 624 & 2 & 70 & 31 & 102 \\
\hline $451 b 5$ & & -31 & 527 & 2 & 59 & 43 & 216 \\
\hline $451 b 6$ & 32 & & 596 & 2 & 78 & 50 & 138 \\
\hline $451 b^{2} 7$ & 36 & & 490 & 2 & 103 & 57 & 253 \\
\hline $451 b 8$ & 37. & $5-38$ & 426 & 2 & 142 & 58 & 398 \\
\hline $451 b 9$ & 42. & & 626 & 2 & 73 & 43 & 171 \\
\hline 452 & 51. & $8-52.3$ & 648 & 2 & 75 & 36 & 107 \\
\hline $451 \mathrm{~b} 10$ & 57 & & 461 & 2 & 121 & 63 & 383 \\
\hline $451 b 11$ & & -73 & 502 & 2 & 108 & 41 & 302 \\
\hline $451 b 12$ & 76. & $5-70$ & 584 & 2 & 93 & 31 & 199 \\
\hline $452 \mathrm{bl}$ & 3 & -6 & 457 & 2 & 89 & 36 & 226 \\
\hline $452 \mathrm{~b} 2$ & 6. & $5-7$ & 311 & 2 & 127 & 53 & 449 \\
\hline $452 \mathrm{~b} 3$ & 11 & -12 & 740 & 2 & 83 & 36 & 75 \\
\hline
\end{tabular}


Table 2. Analytical results for total metal concentration of -80 fraction of samples from holes in the Pinal Creek aquifer, Globe, Arizona--(Continued)

\begin{tabular}{|c|c|c|c|c|c|c|c|}
\hline Sample & $\begin{array}{l}\text { Dep } \\
\text { (fe }\end{array}$ & th & $\begin{array}{c}\mathrm{Ba} \\
\mathrm{mg} / \mathrm{kg}\end{array}$ & $\begin{array}{c}\mathrm{Be} \\
\mathrm{mg} / \mathrm{kg}\end{array}$ & $\begin{array}{c}\mathrm{Ce} \\
\mathrm{mg} / \mathrm{kg}\end{array}$ & $\begin{array}{c}\mathrm{Co} \\
\mathrm{mg} / \mathrm{kg}\end{array}$ & $\begin{array}{l}\mathrm{Cr} \\
\mathrm{mg} / \mathrm{kg}\end{array}$ \\
\hline 452 & 14.8 & -15 & 657 & 2 & 76 & 23 & 49 \\
\hline $452 \mathrm{~b} 4$ & 18 & -19 & 597 & 2 & 75 & 35 & 162 \\
\hline $452 b 5$ & 19 & -21 & 495 & 2 & 108 & 45 & 255 \\
\hline $452 \mathrm{~b} 6$ & 23 & -23 & 638 & 2 & 64 & 51 & 285 \\
\hline 010b1 & 5 & -10 & 490 & 2 & 109 & 29 & 214 \\
\hline $010 \mathrm{~b} 2$ & 15 & -20 & 498 & 2 & 95 & 27 & 209 \\
\hline $010 b 3$ & 23 & -25 & 665 & 2 & 95 & 22 & 144 \\
\hline $010 \mathrm{~b} 4$ & 29 & -30 & 476 & 2 & 118 & 31 & 208 \\
\hline $010 b 5$ & 37 & -38 & 565 & 2 & 95 & 23 & 145 \\
\hline $010 b 6$ & 45 & -46 & 630 & 2 & 96 & 24 & 168 \\
\hline 010b7 & 50 & -52 & 650 & 2 & 105 & 20 & 122 \\
\hline $010 b 8$ & 57.5 & -60 & 589 & 2 & 82 & 19 & 119 \\
\hline 010b9 & 60 & -63 & 546 & 2 & 142 & 15 & 74 \\
\hline 010b10 & 63 & -67 & 567 & 2 & 93 & 25 & 79 \\
\hline 010b11 & 70 & -75 & 506 & 2 & 72 & 16 & 67 \\
\hline 010b12 & 86 & -90 & 515 & 2 & 97 & 17 & 93 \\
\hline
\end{tabular}


Table 2. Analytical results for total metal concentration of -80 fraction of samples from holes in the Pinal Creek aquifer, Globe, Arizona--(Continued)

\begin{tabular}{|c|c|c|c|c|c|c|c|}
\hline Sample & & $\begin{array}{l}\text { pth } \\
\text { eet) }\end{array}$ & $\begin{array}{c}\mathrm{Cu} \\
\mathrm{mg} / \mathrm{kg}\end{array}$ & $\begin{array}{c}\mathrm{Eu} \\
\mathrm{mg} / \mathrm{kg}\end{array}$ & $\begin{array}{c}\mathrm{Ga} \\
\mathrm{mg} / \mathrm{kg}\end{array}$ & $\begin{array}{l}\mathrm{La} \\
\mathrm{mg} / \mathrm{kg}\end{array}$ & $\begin{array}{l}\mathrm{Li} \\
\mathrm{mg} / \mathrm{kg}\end{array}$ \\
\hline$x 1-107 b 1$ & 16 & & 95 & 3 & 22 & 62 & 30 \\
\hline$x 1-107 b 2$ & 17 & & 299 & 2 & 21 & 40 & 42 \\
\hline$x 1-107 b 3$ & 17. & & 2,010 & 2 & 21 & 41 & 38 \\
\hline$x 1-107 b 4$ & 18 & & 320 & $<2$ & 21 & 35 & 29 \\
\hline$x 1-107 b 5$ & 19 & -24 & 292 & 2 & 19 & 43 & 30 \\
\hline$x 1-107 b 6$ & 38 & -39 & 252 & 2 & 21 & 38 & 33 \\
\hline$x 1-107 b 7$ & 46 & -47 & 238 & 2 & 22 & 42 & 29 \\
\hline$x 1-107 b 8$ & 56 & -57 & 211 & 2 & 21 & 43 & 30 \\
\hline$x 1-107 b 9$ & 71 & -72 & 274 & $<2$ & 21 & 42 & 29 \\
\hline$x 5-505 b 1$ & & $7-\quad 1.1$ & 502 & 3 & 10 & 68 & 25 \\
\hline$x 5-505 b 2$ & & $1-\quad 2$ & 212 & 3 & 21 & 76 & 20 \\
\hline$x 5-505 b 3$ & 9 & -10 & 77 & 2 & 19 & 40 & 31 \\
\hline$x 5-505 b 4$ & 10 & -11.5 & 99 & 2 & 21 & 41 & 38 \\
\hline$x 5-505 b 5$ & 18 & -21.5 & 57 & 3 & 19 & 48 & 27 \\
\hline$x 5-505 b 6$ & 23 & -24.8 & 86 & 3 & 19 & 56 & 35 \\
\hline$x 5-505 b 7$ & 58 & -60 & 79 & 2 & 19 & 40 & 43 \\
\hline$x 5-505 b 8$ & 60 & -61.5 & 73 & 2 & 19 & 42 & 36 \\
\hline$x 5-505 b 9$ & 64 & -65 & 44 & $<2$ & 18 & 35 & 41 \\
\hline$x 5-505 b 10$ & 71 & -73 & 34 & $<2$ & 15 & 29 & 33 \\
\hline$x 5-506 b 1$ & & $8-\quad 1.2$ & 577 & 3 & 22 & 56 & 24 \\
\hline$x 5-506 b 2$ & 10. & $5-11.5$ & 75 & 2 & 20 & 45 & 46 \\
\hline$x 5-506 b 3$ & 19 & -22 & 65 & 2 & 19 & 34 & 35 \\
\hline$x 5-506$ & 23 & -24.8 & 96 & 3 & 19 & 46 & 38 \\
\hline $451 \mathrm{~b} 1$ & 10. & & 74 & 3 & 21 & 62 & 34 \\
\hline $451 \mathrm{~b} 2$ & 11 & & 74 & 3 & 20 & 46 & 32 \\
\hline $451 \mathrm{b3}$ & 12 & & 91 & 3 & 22 & 51 & 39 \\
\hline $451 b 4$ & 13 & & 283 & 2 & 19 & 49 & 28 \\
\hline 451 & 21 & & 69 & 2 & 19 & 36 & 36 \\
\hline $451 b 5$ & 30 & -31 & 75 & 2 & 21 & 34 & 33 \\
\hline $451 \mathrm{~b} 6$ & 32 & & 114 & 2 & 20 & 39 & 36 \\
\hline $451 \mathrm{~b} 7$ & 36 & & 89 & 3 & 20 & 57 & 37 \\
\hline $451 \mathrm{~b} 8$ & 37. & $5-38$ & 181 & 3 & 25 & 70 & 33 \\
\hline $451 b 9$ & 42. & & 152 & 2 & 21 & 38 & 34 \\
\hline 451 & 51. & $8-52.3$ & 227 & 2 & 19 & 40 & 35 \\
\hline $451 \mathrm{~b} 10$ & 57 & & 258 & 3 & 22 & 71 & 29 \\
\hline $451 b 11$ & 70 & -73 & 192 & 3 & 21 & 62 & 32 \\
\hline $451 \mathrm{~b} 12$ & 76. & $5-70$ & 246 & 2 & 20 & 48 & 35 \\
\hline $452 \mathrm{bl}$ & 3 & -6 & 111 & 3 & 20 & 48 & 27 \\
\hline $452 \mathrm{~b} 2$ & & $5-7$ & 55 & 3 & 26 & 79 & 19 \\
\hline
\end{tabular}


Table 2. Analytical results for total metal concentration of -80 fraction of samples from holes in the Pinal Creek aquifer, Globe, Arizona--(Continued)

\begin{tabular}{|c|c|c|c|c|c|c|c|}
\hline Sample & Dep & pth & $\mathrm{Cu}$ & Eu & $G a$ & Ia & $\mathrm{Ii}$ \\
\hline $452 \mathrm{~b} 3$ & 11 & -12 & 83 & 2 & 20 & 43 & 39 \\
\hline 452 & 14.8 & $3-15$ & 86 & 2 & 22 & 38 & 55 \\
\hline $452 \mathrm{~b} 4$ & 18 & -19 & 72 & 2 & 20 & 39 & 34 \\
\hline $452 \mathrm{~b} 5$ & 19 & -21 & 83 & 3 & 20 & 60 & 32 \\
\hline $452 \mathrm{~b} 6$ & 23 & -23 & 92 & 3 & 22 & 39 & 31 \\
\hline 010b1 & 5 & -10 & 69 & 3 & 22 & 53 & 26 \\
\hline $010 \mathrm{~b} 2$ & 15 & -20 & 50 & 2 & 19 & 47 & 23 \\
\hline $010 \mathrm{~b} 3$ & 23 & -25 & 56 & 2 & 21 & 48 & 29 \\
\hline $010 \mathrm{~b} 4$ & 29 & -30 & 60 & 3 & 23 & 59 & 26 \\
\hline $010 b 5$ & 37 & -38 & 57 & 2 & 21 & 47 & 30 \\
\hline $010 b 6$ & 45 & -46 & 68 & 2 & 21 & 48 & 28 \\
\hline 010b7 & 50 & -52 & 48 & 2 & 19 & 53 & 29 \\
\hline $010 b 8$ & 57.5 & $5-60$ & 63 & $<2$ & 19 & 42 & 27 \\
\hline $010 b 9$ & 60 & -63 & 27 & 2 & 15 & 66 & 21 \\
\hline $010 b 10$ & 63 & -67 & 36 & $<2$ & 18 & 47 & 31 \\
\hline $010 b 11$ & 70 & -75 & 43 & $<2$ & 18 & 41 & 32 \\
\hline $010 b 12$ & 86 & -90 & 44 & 2 & 18 & 50 & 29 \\
\hline
\end{tabular}


Table 2. Analytical results for total metal concentration of -80 fraction of samples from holes in the Pinal Creek aquifer, Globe, Arizona--(Continued)

\begin{tabular}{|c|c|c|c|c|c|c|c|}
\hline Sample & Dept & & $\begin{array}{c}\text { Mo } \\
\mathrm{mg} / \mathrm{kg}\end{array}$ & $\begin{array}{c}\mathrm{Nb} \\
\mathrm{mg} / \mathrm{kg}\end{array}$ & $\begin{array}{c}\mathrm{Nd} \\
\mathrm{mg} / \mathrm{kg}\end{array}$ & $\begin{array}{c}\mathrm{Ni} \\
\mathrm{mg} / \mathrm{kg}\end{array}$ & $\begin{array}{c}\mathrm{Pb} \\
\mathrm{mg} / \mathrm{kg}\end{array}$ \\
\hline$x 1-107 b 1$ & 16 & & $<2$ & 12 & 60 & 48 & 23 \\
\hline$x 1-107 b 2$ & 17 & & $<2$ & 8 & 40 & 47 & 23 \\
\hline$x 1-107 b 3$ & 17.5 & & 22 & 10 & 41 & 109 & 25 \\
\hline$x 1-107 b 4$ & 18 & & 2 & 8 & 33 & 31 & 28 \\
\hline$x 1-107 b 5$ & 19 & -24 & $<2$ & 12 & 43 & 32 & 22 \\
\hline$x 1-107 b 6$ & 38 & -39 & 3 & 9 & 39 & 36 & 31 \\
\hline$x 1-107 b 7$ & 46 & -47 & $<2$ & 13 & 44 & 38 & 23 \\
\hline$x 1-107 b 8$ & 56 & -57 & $<2$ & 13 & 44 & 39 & 30 \\
\hline$\times 1-107 b 9$ & 71 & -72 & 2 & 12 & 43 & 41 & 40 \\
\hline$x 5-505 b l$ & 0.7 & $-\quad 1.1$ & 16 & 17 & 64 & 163 & 47 \\
\hline$x 5-505 b 2$ & $1.1-$ & -2 & 6 & 22 & 69 & 73 & 45 \\
\hline$x 5-505 b 3$ & $9-$ & -10 & $<2$ & 13 & 40 & 47 & 27 \\
\hline$x 5-505 b 4$ & 10 & -11.5 & 4 & 10 & 39 & 58 & 29 \\
\hline$x 5-505 b 5$ & 18 & -21.5 & $<2$ & 19 & 47 & 50 & 27 \\
\hline$x 5-505 b 6$ & 23 & -24.8 & $<2$ & 19 & 59 & 53 & 28 \\
\hline$x 5-505 b 7$ & 58 & -60 & $<2$ & 13 & 40 & 51 & 29 \\
\hline$x 5-505 b 8$ & 60 & -61.5 & $<2$ & 13 & 41 & 52 & 32 \\
\hline$x 5-505 b 9$ & 64 & -65 & $<2$ & 10 & 32 & 35 & 26 \\
\hline$x 5-505 b 10$ & 71 & -73 & $<2$ & 7 & 25 & 27 & 20 \\
\hline$x 5-506 b 1$ & 0.8 & $-\quad 1.2$ & 21 & 18 & 53 & 200 & 37 \\
\hline$x 5-506 b 2$ & 10.5 & -11.5 & $<2$ & 11 & 47 & 48 & 32 \\
\hline$x 5-506 b 3$ & 19 & -22 & $<2$ & 11 & 36 & 49 & 29 \\
\hline$x 5-506$ & 23 & -24.8 & $<2$ & 16 & 49 & 54 & 29 \\
\hline $451 \mathrm{bl}$ & 10.5 & & $<2$ & 15 & 58 & 66 & 31 \\
\hline $451 b 2$ & 11 & & $<2$ & 15 & 48 & 55 & 29 \\
\hline $451 \mathrm{b3}$ & 12 & & 2 & 15 & 53 & 58 & 36 \\
\hline $451 b 4$ & 13 & 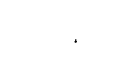 & $<2$ & 11 & 44 & 34 & 20 \\
\hline 451 & 21 & & $<2$ & 8 & 38 & 52 & 26 \\
\hline $451 b 5$ & 30 & -31 & $<2$ & 19 & 36 & 58 & 29 \\
\hline $451 \mathrm{~b} 6$ & 32 & & $<2$ & 12 & 40 & 60 & 33 \\
\hline $451 b_{7}$ & 36 & & $<2$ & 20 & 59 & 66 & 36 \\
\hline $451 b 8$ & 37.5 & -38 & $<2$ & 26 & 69 & 73 & 43 \\
\hline $451 b 9$ & 42.5 & & $<2$ & 14 & 37 & 58 & 42 \\
\hline 451 & 51.8 & -52.3 & $<2$ & 10 & 38 & 49 & 31 \\
\hline $451 b 10$ & 57 & & $<2$ & 27 & 71 & 70 & 32 \\
\hline $451 b 11$ & 70 & -73 & $<2$ & 24 & 59 & 62 & 27 \\
\hline $451 b 12$ & 76.5 & -70 & $<2$ & 13 & 50 & 53 & 26 \\
\hline $452 \mathrm{~b} 1$ & 3. & -6 & $<2$ & 17 & 52 & 54 & 44 \\
\hline $452 \mathrm{~b} 2$ & 6.5 & -7 & $<2$ & 28 & 79 & 65 & 28 \\
\hline $452 \mathrm{~b} 3$ & 11 & -12 & $<2$ & 9 & 43 & 54 & 37 \\
\hline
\end{tabular}


Table 2. Analytical results for total metal concentration of -80 fraction of samples from holes in the Pinal Creek aquifer, Globe, Arizona--(Continued)

\begin{tabular}{|c|c|c|c|c|c|c|c|}
\hline Sample & $\begin{array}{l}\text { Dept } \\
\text { lfer }\end{array}$ & eth & $\begin{array}{c}\text { Mo } \\
\mathrm{mg} / \mathrm{kg}\end{array}$ & $\begin{array}{c}\mathrm{Nb} \\
\mathrm{mg} / \mathrm{kg}\end{array}$ & $\begin{array}{l}\mathrm{Nd} \\
\mathrm{mg} / \mathrm{kg}\end{array}$ & $\begin{array}{c}\mathrm{Ni} \\
\mathrm{mg} / \mathrm{kg}\end{array}$ & $\begin{array}{c}\mathrm{Pb} \\
\mathrm{mg} / \mathrm{kg}\end{array}$ \\
\hline 452 & 14.8 & $3-15$ & $<2$ & 8 & 36 & 53 & 30 \\
\hline $452 b 4$ & 18 & -19 & $<2$ & 14 & 42 & 57 & 29 \\
\hline $452 b 5$ & 19 & -21 & $<2$ & 22 & 62 & 65 & 39 \\
\hline $452 \mathrm{~b} 6$ & 23 & -23 & $<2$ & 21 & 44 & 64 & 27 \\
\hline 010b1 & 5. & -10 & $<2$ & 8 & 58 & 59 & 24 \\
\hline $010 \mathrm{~b} 2$ & 15 & -20 & $<2$ & 8 & 49 & 68 & 19 \\
\hline $010 \mathrm{~b} 3$ & 23 & -25 & 3 & 15 & 47 & 86 & 23 \\
\hline $010 \mathrm{~b} 4$ & 29 & -30 & $<2$ & 8 & 61 & 59 & 20 \\
\hline $010 \mathrm{~b} 5$ & 37 & -38 & $<2$ & 7 & 48 & 54 & 23 \\
\hline $010 b 6$ & 45 & -46 & 2 & 8 & 49 & 70 & 26 \\
\hline $010 \mathrm{~b} 7$ & 50 & -52 & 4 & 6 & 49 & 102 & 22 \\
\hline 010b8 & 57.5 & $5-60$ & $<2$ & 7 & 40 & 59 & 20 \\
\hline 010b9 & 60 & -63 & $<2$ & 6 & 73 & 25 & 19 \\
\hline $010 b 10$ & 63 & -67 & $<2$ & 5 & 46 & 41 & 31 \\
\hline 010b11 & 70 & -75 & $<2$ & 5 & 35 & 38 & 21 \\
\hline 010b12 & 86 & -90 & $<2$ & 5 & 46 & 42 & 23 \\
\hline
\end{tabular}


Table 2. Analytical results for total metal concentration of -80 fraction of samples from holes in the Pinal Creek aquifer, Globe, Arizona--(Continued)

\begin{tabular}{|c|c|c|c|c|c|c|c|}
\hline Sample & $\begin{array}{l}\text { Dept) } \\
\text { (feet }\end{array}$ & & $\begin{array}{c}\mathrm{sc} \\
\mathrm{mg} / \mathrm{kg}\end{array}$ & $\begin{array}{c}\mathrm{sr} \\
\mathrm{mg} / \mathrm{kg}\end{array}$ & $\begin{array}{c}\mathrm{Th} \\
\mathrm{mg} / \mathrm{kg}\end{array}$ & $\begin{array}{c}\mathrm{V} \\
\mathrm{mg} / \mathrm{kg}\end{array}$ & $\frac{\mathrm{Y}}{\mathrm{mg} / \mathrm{kg}}$ \\
\hline$x 1-107 b 1$ & 16 & & 24 & 339 & 14 & 223 & 32 \\
\hline$x 1-107 b 2$ & 17 & & 18 & 283 & 10 & 119 & 28 \\
\hline$x 1-107 b 3$ & 17.5 & & 16 & 298 & 17 & 119 & 31 \\
\hline$x 1-107 b 4$ & 18 & & 14 & 325 & 10 & 123 & 24 \\
\hline$x 1-107 b 5$ & $19-$ & -24 & 17 & 335 & 10 & 162 & 28 \\
\hline$x 1-107 b 6$ & $38-$ & -39 & 17 & 310 & 12 & 148 & 28 \\
\hline$x 1-107 b 7$ & $46-$ & -47 & 17 & 301 & 14 & 232 & 30 \\
\hline$x 1-107 b 8$ & $56-$ & -57 & 18 & 308 & 20 & 242 & 28 \\
\hline$x 1-107 b 9$ & $71-$ & 72 & 16 & 279 & 11 & 263 & 24 \\
\hline$x 5-505 b 1$ & $0.7-$ & 1.1 & 18 & 260 & 30 & 334 & 30 \\
\hline$x 5-505 b 2$ & $1.1-$ & 2 & 19 & 230 & 17 & 551 & 28 \\
\hline$x 5-505 b 3$ & $9-$ & 10 & 18 & 270 & 11 & 251 & 26 \\
\hline$x 5-505 b 4$ & $10-$ & 11.5 & 18 & 301 & 11 & 156 & 28 \\
\hline$x 5-505 b 5$ & $18-$ & 21.5 & 21 & 282 & 11 & 383 & 28 \\
\hline$x 5-505 b 6$ & $23-$ & 24.8 & 22 & 279 & 22 & 330 & 41 \\
\hline$x 5-505 b 7$ & $58-$ & 60 & 18 & 272 & 18 & 219 & 31 \\
\hline$x 5-505 b 8$ & $60-$ & 61.5 & 18 & 264 & 27 & 281 & 29 \\
\hline$x 5-505 b 9$ & $64-$ & 65 & 16 & 610 & 8 & 121 & 25 \\
\hline$x 5-505 b 10$ & $71-$ & 73 & 10 & 643 & 7 & 70 & 18 \\
\hline$x 5-506 b 1$ & $0.8-$ & - $\quad 1.2$ & 18 & 225 & 35 & 405 & 30 \\
\hline$x 5-506 b 2$ & $10.5-$ & 11.5 & 20 & 271 & 33 & 178 & 33 \\
\hline$x 5-506 b 3$ & $19-$ & -22 & 18 & 283 & 19 & 179 & 27 \\
\hline$x 5-506$ & $23-$ & -24.8 & 22 & 288 & 18 & 251 & 36 \\
\hline $451 b 1$ & 10.5 & & 22 & 275 & 16 & 329 & 34 \\
\hline $451 \mathrm{~b} 2$ & 11 & & 20 & 284 & 13 & 282 & 33 \\
\hline $451 b 3$ & 12 & & 21 & 281 & 12 & 266 & 33 \\
\hline $451 \mathrm{~b} 4$ & 13 & 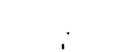 & 17 & 334 & 11 & 178 & 30 \\
\hline 451 & 21 & & 18 & 265 & 11 & 195 & 24 \\
\hline $451 b 5$ & $30-31$ & & 20 & 262 & 9 & 403 & 26 \\
\hline $451 b 6$ & 32 & & 20 & 289 & 9 & 247 & 27 \\
\hline $451 b 7$ & 36 & & 22 & 239 & 19 & 412 & 39 \\
\hline $451 b 8$ & $37.5-$ & 38 & 24 & 206 & 30 & 684 & 39 \\
\hline $451 b 9$ & 42.5 & & 22 & 279 & 9 & 323 & 27 \\
\hline 451 & $51.8-$ & -52.3 & 18 & 282 & 9 & 189 & 26 \\
\hline $451 b 10$ & 57 & & 25 & 223 & 25 & 713 & 36 \\
\hline $451 b 11$ & $70-$ & 73 & 24 & 248 & 20 & 529 & 33 \\
\hline $451 b 12$ & $76.5-$ & 70 & 17 & 245 & 12 & 304 & 30 \\
\hline $452 \mathrm{bl}$ & $3-$ & - 6 & 20 & 258 & 17 & 410 & 35 \\
\hline $452 \mathrm{~b} 2$ & $6.5-$ & 7 & 24 & 193 & 41 & 845 & 36 \\
\hline $452 \mathrm{b3}$ & $11-$ & 12 & 17 & 277 & 20 & 136 & 28 \\
\hline
\end{tabular}


Table 2. Analytical results for total metal concentration of -80 fraction of samples from holes in the Pinal Creek aquifer, Globe, Arizona-m(Continued)

\begin{tabular}{|c|c|c|c|c|c|c|c|}
\hline Sample & $\begin{array}{l}\text { De } \\
\text { lf }\end{array}$ & $\begin{array}{l}\text { pth } \\
\text { eet) }\end{array}$ & $\begin{array}{c}\mathrm{sc} \\
\mathrm{mg} / \mathrm{kg}\end{array}$ & $\begin{array}{c}\mathrm{Sr} \\
\mathrm{mg} / \mathrm{kg}\end{array}$ & $\begin{array}{c}\mathrm{Th} \\
\mathrm{mg} / \mathrm{kg}\end{array}$ & $\begin{array}{c}\mathrm{V} \\
\mathrm{mg} / \mathrm{kg}\end{array}$ & $\begin{array}{c}\mathrm{Y} \\
\mathrm{mg} / \mathrm{kg}\end{array}$ \\
\hline 452 & 14.8 & $8-15$ & 19 & 231 & 10 & 97 & 23 \\
\hline $452 \mathrm{~b} 4$ & 18 & -19 & 20 & 278 & 10 & 283 & 27 \\
\hline $452 b 5$ & 19 & -21 & 24 & 270 & 28 & 464 & 37 \\
\hline $452 b 6$ & 23 & -23 & 21 & 262 & 11 & 506 & 29 \\
\hline $010 \mathrm{~b} 1$ & 5 & -10 & 26 & 330 & 12 & 273 & 34 \\
\hline 010b2 & 15 & -20 & 21 & 286 & 11 & 245 & 26 \\
\hline $010 b 3$ & 23 & -25 & 17 & 248 & 14 & 149 & 23 \\
\hline $010 b 4$ & 29 & -30 & 24 & 316 & 14 & 271 & 33 \\
\hline $010 b 5$ & 37 & -38 & 20 & 274 & 12 & 189 & 27 \\
\hline $010 b 6$ & 45 & -46 & 21 & 249 & 11 & 193 & 25 \\
\hline $010 \mathrm{~b} 7$ & 50 & -52 & 16 & 223 & 15 & 120 & 20 \\
\hline $010 b 8$ & 57 & $5-60$ & 16 & 226 & 11 & 133 & 22 \\
\hline 010b9 & 60 & -63 & 11 & 184 & 18 & 88 & 21 \\
\hline 010b10 & 63 & -67 & 13 & 162 & 11 & 93 & 20 \\
\hline $010 \mathrm{~b} 11$ & 70 & -75 & 13 & 188 & 8 & 92 & 19 \\
\hline $010 \mathrm{~b} 12$ & 86 & -90 & 14 & 196 & 9 & 114 & 22 \\
\hline
\end{tabular}


Table 2. Analytical results for total metal concentration of -80 fraction of samples from holes in the Pinal Creek aquifer, Globe, Arizona--(Continued)

\begin{tabular}{|c|c|c|c|c|}
\hline Sample & $\begin{array}{l}\text { Dept) } \\
\text { (fee }\end{array}$ & & $\begin{array}{c}\mathrm{Yb} \\
\mathrm{mg} / \mathrm{kg}\end{array}$ & $\begin{array}{c}2 \mathrm{n} \\
\mathrm{mg} / \mathrm{kg}\end{array}$ \\
\hline$x 1-107 b 1$ & 16 & & 4 & 101 \\
\hline$x 1-107 b 2$ & 17 & & 3 & 123 \\
\hline$x 1-107 b 3$ & 17.5 & & 3 & 163 \\
\hline$x 1-107 b 4$ & 18 & & 3 & 80 \\
\hline$x 1-107 b 5$ & $19-$ & - 24 & 3 & 88 \\
\hline$x 1-107 b 6$ & $38-$ & 39 & 3 & 103 \\
\hline$x 1-107 b 7$ & $46-$ & 47 & 3 & 107 \\
\hline$x 1-107 b 8$ & $56-$ & -57 & 4 & 105 \\
\hline x1-107b9 & $71-$ & - 72 & 3 & 117 \\
\hline$x 5-505 b 1$ & $0.7-$ & . 1.1 & 4 & 306 \\
\hline$x 5-505 b 2$ & $1.1-$ & 2 & 4 & 231 \\
\hline$x 5-505 b 3$ & $9-$ & - 10 & 4 & 141 \\
\hline$x 5-505 b 4$ & $10-$ & 11.5 & 4 & 130 \\
\hline$x 5-505 b 5$ & $18-$ & .21 .5 & 4 & 158 \\
\hline$x 5-505 b 6$ & $23-$ & . 24.8 & 5 & 162 \\
\hline$x 5-505 b 7$ & $58-$ & 60 & 4 & 129 \\
\hline$x 5-505 b 8$ & $60-$ & 61.5 & 4 & 142 \\
\hline$x 5-505 b 9$ & $64-$ & - 65 & 3 & 88 \\
\hline$x 5-505 b 10$ & $71-$ & 73 & 2 & 61 \\
\hline$x 5-506 b 1$ & $0.8-$ & -1.2 & 4 & 322 \\
\hline$x 5-506 b 2$ & $10.5-$ & 11.5 & 4 & 146 \\
\hline$x 5-506 b 3$ & $19-$ & -22 & 4 & 131 \\
\hline$x 5-506$ & $23-$ & $=24.8$ & 5 & 159 \\
\hline $451 b 1$ & 10.5 & & 5 & 182 \\
\hline $451 \mathrm{~b} 2$ & 11 & & 4 & 165 \\
\hline $451 b 3$ & 12 & & 4 & 177 \\
\hline $451 b 4$ & 13 & . & 3 & 87 \\
\hline 451 & 21 & & 3 & 173 \\
\hline $451 \mathrm{~b} 5$ & $30-$ & -31 & 3 & 225 \\
\hline $451 b 6$ & 32 & & 4 & 207 \\
\hline $451 b 7$ & 36 & & 5 & 293 \\
\hline $451 b 8$ & $37.5-$ & 38 & 5 & 286 \\
\hline $451 \mathrm{bg}$ & 42.5 & & 4 & 215 \\
\hline 451 & $51.8-$ & 52.3 & 3 & 152 \\
\hline $451 b 10$ & 57 & & 5 & 262 \\
\hline $451 b 11$ & $70-$ & 73 & 5 & 204 \\
\hline $451 b 12$ & $76.5-$ & 70 & 3 & 155 \\
\hline $452 \mathrm{bl}$ & $3-$ & 6 & 4 & 172 \\
\hline $452 \mathrm{b2}$ & $6.5-$ & 7 & 5 & 239 \\
\hline $452 b 3$ & $11-$ & 12 & 3 & 156 \\
\hline
\end{tabular}


Table 2. Analytical results for total metal concentration of -80 fraction of samples from holes in the Pinal Creek aquifer, Globe, Arizona--(Continued)

\begin{tabular}{|c|c|c|c|c|}
\hline Sample & $\begin{array}{l}\text { Dept } \\
\text { lfee }\end{array}$ & $\begin{array}{l}\text { th } \\
\text { et) }\end{array}$ & $\begin{array}{c}\mathrm{Yb} \\
\mathrm{mg} / \mathrm{kg}\end{array}$ & $\begin{array}{c}\mathrm{zn} \\
\mathrm{mg} / \mathrm{kg}\end{array}$ \\
\hline 452 & $14.8-$ & -15 & 3 & 140 \\
\hline $452 \mathrm{~b} 4$ & 18 & -19 & 4 & 179 \\
\hline $452 \mathrm{~b} 5$ & 19 & -21 & 5 & 230 \\
\hline $452 b 6$ & 23 & -23 & 4 & 224 \\
\hline 010b1 & 5 & -10 & 4 & 121 \\
\hline $010 \mathrm{~b} 2$ & 15 & -20 & 3 & 101 \\
\hline $010 b 3$ & 23 & -25 & 3 & 108 \\
\hline $010 b 4$ & 29 & -30 & 3 & 97 \\
\hline $010 b 5$ & 37 & -38 & 3 & 106 \\
\hline $010 b 6$ & 45 & -46 & 3 & 116 \\
\hline $010 b 7$ & 50 & -52 & 2 & 98 \\
\hline $010 b 8$ & 57.5 & -60 & 2 & 102 \\
\hline 010b9 & 60 & -63 & 2 & 55 \\
\hline 010b10 & 63 & -67 & 2 & 89 \\
\hline 010b11 & 70 & -75 & 2 & 98 \\
\hline 010b12 & 86 & -90 & 2 & 100 \\
\hline
\end{tabular}


Table 3. Analytical results for partial sequential extractions of Pinal Creek aquifer material.

1--, no data available; $\mathrm{Cu}(\mathrm{carb}), \mathrm{Zn}(\mathrm{carb})$, etc. represents the result for the carbonate extraction; $\mathrm{Cu}(\mathrm{MnO}), \mathrm{Zn}(\mathrm{MnO})$, etc. the result for the manganese

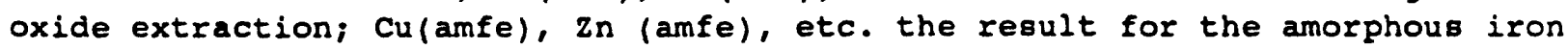
oxide extraction; Cu(cryfe) the result for the crystalline iron oxide extraction; and $\mathrm{Cu}(\mathrm{res})$ the result for the residual digestion]

\begin{tabular}{|c|c|c|c|c|c|c|c|}
\hline Sample & \multicolumn{2}{|c|}{$\begin{array}{l}\text { Depth } \\
\text { (feet) }\end{array}$} & $\begin{array}{l}\mathrm{Cu}(\mathrm{carb}) \\
\mathrm{mg} / \mathrm{kg}\end{array}$ & $\begin{array}{l}\mathrm{Cu}(\mathrm{MnO}) \\
\mathrm{mg} / \mathrm{kg}\end{array}$ & $\begin{array}{l}\text { Cu (amfe) } \\
\mathrm{mg} / \mathrm{kg}\end{array}$ & $\begin{array}{l}\mathrm{Cu}(\mathrm{cryfe}) \\
\mathrm{mg} / \mathrm{kg}\end{array}$ & $\begin{array}{l}\text { Cu (res) } \\
\mathrm{mg} / \mathrm{kg}\end{array}$ \\
\hline$x 1-107 b 1$ & \multicolumn{2}{|l|}{16} & 7.5 & 3.1 & 18 & 17 & 39 \\
\hline$x 1-107 b 2$ & \multicolumn{2}{|l|}{17} & 73 & 2.5 & 106 & 69 & 48 \\
\hline$x 1-107 b 3$ & \multicolumn{2}{|c|}{17.5} & 536 & 780 & 311 & 31 & 67 \\
\hline$x 1-107 b 4$ & \multicolumn{2}{|c|}{18} & 108 & 4.1 & 104 & 50 & 72 \\
\hline$x 1-107 b 5$ & 19 & -24 & 94 & 9.2 & 66 & 28 & 53 \\
\hline$x 1-107 b 6$ & 38 & -39 & 60 & 1.4 & 36 & 85 & 43 \\
\hline$x 1-107 b 7$ & 46 & -47 & 83 & 1.7 & 29 & 32 & 98 \\
\hline$x 1-107 b 8$ & 56 & -57 & 55 & 1.9 & 21 & 81 & 37 \\
\hline$x 1-107 b 9$ & 71 & -72 & 93 & 2.6 & 22 & 75 & 64 \\
\hline$x 5-505 b 1$ & \multicolumn{2}{|c|}{$0.7-1.1$} & 15 & 165 & 159 & 49 & 50 \\
\hline$x 5-505 b 2$ & \multicolumn{2}{|c|}{$1.1-2$} & 20 & 26 & 53 & 50 & 59 \\
\hline$x 5-505 b 3$ & 9 & -10 & 8.8 & 4.2 & 18 & 18 & 17 \\
\hline$x 5-505 b 4$ & 10 & -11.5 & 9.0 & 10 & 20 & 22 & 22 \\
\hline$x 5-505 b 5$ & 18 & -21.5 & 4.5 & 1.5 & 10 & 20 & 17 \\
\hline$x 5-505 b 6$ & 23 & -24.8 & 4.5 & 1.8 & 20 & 27 & 27 \\
\hline$x 5-505 b 7$ & 58 & -60 & 8.0 & 5.2 & 20 & 18 & 26 \\
\hline$x 5-505 b 8$ & 60 & -61.5 & 6.4 & 4.0 & 15 & 21 & 23 \\
\hline x5-505b9 & 64 & -65 & 3.9 & 0.9 & 5 & 15 & 18 \\
\hline$x 5-505 b 10$ & 71 & -73 & 4.3 & 0.3 & 3 & 11 & 12 \\
\hline$x 5-506 b 1$ & & $8-1.2$ & 13 & 166 & 187 & 34 & 86 \\
\hline$x 5-506 b 2$ & 10. & $5-11.5$ & 3.9 & 1.8 & 15 & 11 & 38 \\
\hline$x 5-506 b 3$ & 19 & -22 & 17 & 2.4 & 18 & 40 & 17 \\
\hline$x 5-506$ & 23 & -24.8 & 9.2 & 2.9 & 16 & 32 & 29 \\
\hline $451 \mathrm{bl}$ & 10. & & 3.8 & 2.8 & 12 & 14 & 36 \\
\hline $451 \mathrm{~b} 2$ & 11 & & 7.8 & 0.5 & 14 & 29 & 25 \\
\hline $451 \mathrm{~b} 3$ & 12 & & 4.1 & 0.9 & 15 & 20 & 40 \\
\hline $451 b 4$ & 13 & & 72 & 13 & 55 & 26 & 65 \\
\hline 451 & 21 & & 5.0 & 0.9 & 12 & 30 & 24 \\
\hline $451 b 5$ & 30 & -31 & 7.9 & 1.2 & 13 & 25 & 25 \\
\hline $451 b 6$ & 32 & & 15 & 12 & 21 & 14 & 40 \\
\hline $451 b 7$ & 36 & & 7.7 & 0.5 & 13 & 34 & 25 \\
\hline $451 b 8$ & 37. & $5-38$ & 49 & 8.3 & 36 & 16 & 46 \\
\hline $451 b 9$ & 42 . & & 43 & 5.6 & 37 & 34 & 20 \\
\hline 451 & 51. & $8-52.3$ & 80 & 18 & 61 & 18 & 27 \\
\hline $451 \mathrm{~b} 10$ & 57 & & 118 & 13 & 66 & 31 & 19 \\
\hline
\end{tabular}


Table 3. Analytical results for partial sequential extractions of Pinal Creek aquifer material--(Continued)

\begin{tabular}{|c|c|c|c|c|c|c|c|}
\hline \multirow{2}{*}{$\begin{array}{l}\text { Sample } \\
451 b 11\end{array}$} & \multicolumn{2}{|c|}{$\begin{array}{l}\text { Depth } \\
\text { (feet) }\end{array}$} & \multirow{2}{*}{$\frac{\begin{array}{l}\mathrm{Cu}(\mathrm{carb}) \\
\mathrm{mg} / \mathrm{kg}\end{array}}{73}$} & \multirow{2}{*}{$\begin{array}{c}\mathrm{Cu}(\mathrm{MnO}) \\
\mathrm{mg} / \mathrm{kg}\end{array}$} & \multirow{2}{*}{$\frac{\begin{array}{l}\mathrm{Cu}(\mathrm{amfe}) \\
\mathrm{mg} / \mathrm{kg}\end{array}}{42}$} & \multirow{2}{*}{$\begin{array}{l}\mathrm{Cu}(\mathrm{cryfe}) \\
\mathrm{mg} / \mathrm{kg} \\
40\end{array}$} & \multirow{2}{*}{$\begin{array}{l}\mathrm{Cu} \text { (res) } \\
\mathrm{mg} / \mathrm{kg} \\
29\end{array}$} \\
\hline & 70 & -73 & & & & & \\
\hline $451 b 12$ & 76. & $5-70$ & 95 & 2.2 & 35 & 60 & 25 \\
\hline $452 \mathrm{bl}$ & 3 & -6 & 19 & 1.5 & 30 & 30 & 29 \\
\hline $452 \mathrm{~b} 2$ & 6. & $5-7$ & 2.3 & 0.6 & 5.6 & 17 & 26 \\
\hline $452 \mathrm{~b} 3$ & 11 & -12 & 5.2 & 0.8 & 15 & 26 & 32 \\
\hline 452 & 14. & $8-15$ & 5.6 & 0.7 & 15 & 41 & 28 \\
\hline $452 \mathrm{~b} 4$ & 18 & -19 & 3.1 & 0.7 & 11 & 26 & 24 \\
\hline $452 \mathrm{~b} 5$ & 19 & -21 & 4.0 & 0.6 & 13 & 25 & 32 \\
\hline $452 \mathrm{~b} 6$ & 23 & -23 & 11 & 1.1 & 11 & 38 & 31 \\
\hline 010b1 & 5 & -10 & 4.9 & 0.3 & 9.5 & 13 & 48 \\
\hline 010b2 & 15 & -20 & 8.0 & 0.6 & 7.5 & 8.9 & 34 \\
\hline 010b3 & 23 & -25 & 7.3 & 0.8 & 16 & 23 & 15 \\
\hline $010 b 4$ & 29 & -30 & 5.4 & 0.3 & 9.4 & 14 & 45 \\
\hline 010b5 & 37 & -38 & 5.1 & 0.3 & 10 & 31 & 19 \\
\hline $010 \mathrm{~b} 6$ & 45 & -46 & 8.5 & 0.9 & 16 & 32 & 16 \\
\hline $010 b 7$ & 50 & -52 & 8.9 & 0.8 & 9.4 & 20 & 16 \\
\hline $010 \mathrm{~b} 8$ & 57. & $5-60$ & 9.0 & 0.5 & 15 & 24 & 17 \\
\hline $010 b 9$ & 60 & -63 & 7.8 & 0.3 & 9.2 & 20 & 9.2 \\
\hline $010 b 10$ & 63 & -67 & 4.2 & 0.3 & 4.2 & 18 & 18 \\
\hline $010 b 11$ & 70 & -75 & 4.7 & 0.3 & 3.8 & 21 & 23 \\
\hline 010b12 & 86 & -90 & 4.9 & 0.4 & 4.7 & 20 & 25 \\
\hline
\end{tabular}


Table 3. Analytical results for partial sequential extractions of Pinal Creek aquifer material--(Continued)

\begin{tabular}{|c|c|c|c|c|c|c|c|}
\hline Sample & \multicolumn{2}{|c|}{$\begin{array}{l}\text { Depth } \\
\text { (feet) }\end{array}$} & $\begin{array}{l}\mathrm{zn}(\mathrm{carb}) \\
\mathrm{mg} / \mathrm{kg}\end{array}$ & $\begin{array}{l}\mathrm{Zn}(\mathrm{MnO}) \\
\mathrm{mg} / \mathrm{kg}\end{array}$ & $\begin{array}{l}\mathrm{Zn}(\operatorname{amfe}) \\
\mathrm{mg} / \mathrm{kg}\end{array}$ & $\begin{array}{l}\mathrm{Zn}(\operatorname{cryfe}) \\
\mathrm{mg} / \mathrm{kg}\end{array}$ & $\begin{array}{l}\mathrm{zn} \text { (res) } \\
\mathrm{mg} / \mathrm{kg}\end{array}$ \\
\hline$x 1-107 b 1$ & \multicolumn{2}{|l|}{16} & 7.2 & 2.8 & 15 & 22 & 65 \\
\hline$x 1-107 b 2$ & \multicolumn{2}{|l|}{17} & 8.5 & 1.4 & 27 & 46 & 33 \\
\hline$x 1-107 b 3$ & \multicolumn{2}{|c|}{17.5} & 20 & 34 & 22 & 18 & 59 \\
\hline$x 1-107 b 4$ & \multicolumn{2}{|c|}{18} & 7.4 & 0.3 & 16 & 29 & 60 \\
\hline$x 1-107 b 5$ & 19 & -24 & 6.9 & 0.7 & 11 & 24 & 53 \\
\hline$x 1-107 b 6$ & 38 & -39 & 8.0 & 0.3 & 8 & 40 & 46 \\
\hline$x 1-107 b 7$ & 46 & -47 & 8.0 & 0.3 & 12 & 14 & 96 \\
\hline$x 1-107 b 8$ & 56 & -57 & 6.7 & 0.6 & 14 & 52 & 61 \\
\hline$x 1-107 b 9$ & 71 & -72 & 15 & 1.4 & 9 & 31 & 66 \\
\hline$x 5-505 b 1$ & & $7-1.1$ & 20 & 150 & 38 & 37 & 69 \\
\hline$x 5-505 b 2$ & & $1-2$ & 10.0 & 13 & 17 & 28 & 128 \\
\hline$x 5-505 b 3$ & 9 & -10 & 3.4 & 3.0 & 13 & 31 & 62 \\
\hline$x 5-505 b 4$ & 10 & -11.5 & 3.8 & 6.1 & 18 & 38 & 47 \\
\hline$x 5-505 b 5$ & 18 & -21.5 & 2.1 & 2.2 & 13 & 31 & 99 \\
\hline$x 5-505 b 6$ & 23 & -24.8 & 3.9 & 1.9 & 21 & 37 & 80 \\
\hline$x 5-505 b 7$ & 58 & -60 & 2.8 & 2.8 & 17 & 26 & 73 \\
\hline$x 5-505 b 8$ & 60 & -61.5 & 2.5 & 2.5 & 16 & 33 & 85 \\
\hline X5-505b9 & 64 & -65 & 2.1 & 2.7 & 7.9 & 21 & 63 \\
\hline$x 5-505 b 10$ & 71 & -73 & 1.5 & 1.0 & 4.5 & 18 & 27 \\
\hline$x 5-506 b 1$ & & $8-1.2$ & 5.2 & 116 & 36 & 27 & 126 \\
\hline$x 5-506 b 2$ & 10. & $5-11 \cdot 5$ & 4.9 & 1.5 & 20 & 25 & 95 \\
\hline$x 5-506 b 3$ & 19 & -22 & 9.0 & 3.2 & 14 & 48 & 63 \\
\hline$x 5-506$ & 23 & -24.8 & 5.2 & 2.1 & 11 & 33 & 76 \\
\hline $451 b 1$ & 10. & & 9.0 & 5.6 & 20 & 36 & 106 \\
\hline $451 b 2$ & 11 & & 5.6 & 2.5 & 17 & 48 & 70 \\
\hline $451 \mathrm{b3}$ & 12 & & 5.4 & 3.9 & 24 & 40 & 86 \\
\hline $451 b 4$ & 13 & & 5.6 & 1.2 & 8.2 & 15 & 59 \\
\hline 451 & 21 & & 14 & 3.7 & 22 & 56 & 57 \\
\hline $45 I b 5$ & 30 & $-3 ?$ & 18 & 3.7 & 28 & 42 & 111 \\
\hline $451 b 6$ & 32 & & 22 & 8.1 & 34 & 29 & 101 \\
\hline $451 \mathrm{~b} 7$ & 36 & & 42 & 4.6 & 62 & 51 & 108 \\
\hline $451 b 8$ & 37. & $5-38$ & 18 & 5.3 & 26 & 24 & 161 \\
\hline $451 b 9$ & 42. & & 11 & 24.6 & 14 & 59 & 112 \\
\hline 451 & 51. & $8-52.3$ & 12 & 3.4 & 26 & 34 & 69 \\
\hline $451 b 10$ & 57 & & 11 & 2.0 & 18 & 30 & 111 \\
\hline $451 b 11$ & 70 & -73 & 10 & 0.5 & 15 & 35 & 114 \\
\hline $451 \mathrm{~b} 12$ & 76. & $5-70$ & 9.8 & 0.8 & 15 & 45 & 77 \\
\hline $452 \mathrm{bl}$ & 3 & -6 & 9.5 & 3.1 & 10 & 32 & 118 \\
\hline $452 \mathrm{~b} 2$ & & $5-7$ & 1.4 & 1.5 & 6.5 & 26 & 167 \\
\hline $452 b 3$ & 11 & -12 & 5.2 & 4.3 & 20 & 46 & 71 \\
\hline
\end{tabular}


Table 3. Analytical results for partial sequential extractions of Pinal Creek aquifer material--(Continued)

\begin{tabular}{|c|c|c|c|c|c|c|c|}
\hline \multirow{2}{*}{$\begin{array}{l}\text { Sample } \\
452\end{array}$} & \multicolumn{2}{|c|}{$\begin{array}{l}\text { Depth } \\
\text { (feet) }\end{array}$} & \multirow{2}{*}{$\frac{\mathrm{mg}(\text { carb })}{\mathrm{mg} / \mathrm{kg}}$} & \multirow{2}{*}{$\frac{\begin{array}{l}\mathrm{zn}(\mathrm{MnO}) \\
\mathrm{mg} / \mathrm{kg}\end{array}}{0.7}$} & \multirow{2}{*}{$\begin{array}{c}\mathrm{zn}(\operatorname{amfe}) \\
\mathrm{mg} / \mathrm{kg}\end{array}$} & \multirow{2}{*}{$\begin{array}{c}\begin{array}{l}\mathrm{Zn}(\mathrm{cryfe}) \\
\mathrm{mg} / \mathrm{kg}\end{array} \\
78\end{array}$} & \multirow{2}{*}{$\begin{array}{c}\mathrm{zn} \text { (res) } \\
\mathrm{mg} / \mathrm{kg}\end{array}$} \\
\hline & 14. & $8-15$ & & & & & \\
\hline $452 b 4$ & 18 & -19 & 8.0 & 3.1 & 18 & 44 & 63 \\
\hline $452 \mathrm{~b} 5$ & 19 & -21 & 12 & 3.1 & 24 & 37 & 106 \\
\hline $452 \mathrm{~b} 6$ & 23 & -23 & 12 & 4.1 & 20 & 50 & 134 \\
\hline 010b1 & 5 & -10 & 15 & 1.9 & 27 & 28. & 81 \\
\hline $010 \mathrm{~b} 2$ & 15 & -20 & 54 & 2.2 & 13 & 13 & 61 \\
\hline $010 b 3$ & 23 & -25 & 13 & 1.3 & 20 & 41 & 27 \\
\hline $010 \mathrm{~b} 4$ & 29 & -30 & 4.5 & 0.9 & 51 & 30 & 74 \\
\hline 010b5 & 37 & -38 & 8.0 & 0.8 & 11 & 43 & 33 \\
\hline $010 b 6$ & 45 & -46 & 23 & 1.8 & 16 & 41 & 29 \\
\hline $010 b 7$ & 50 & -52 & 13 & 1.9 & 11 & 28 & 33 \\
\hline $010 \mathrm{~b} 8$ & 57. & $5-60$ & 12 & 1.3 & 22 & 37 & 27 \\
\hline 010b9 & 60 & -63 & 5.2 & 1.4 & 8 & 22 & 65 \\
\hline $010 b 10$ & 63 & -67 & 12 & 0.7 & 9 & 39 & 28 \\
\hline 010b11 & 70 & -75 & 25 & 2.0 & 24 & 55 & 35 \\
\hline $010 b 12$ & 86 & -90 & 28 & 2.2 & 8 & 38 & 22 \\
\hline
\end{tabular}


Table 3. Analytical results for partial sequential extractions of Pinal Creek aquifer material--(Continued)

\begin{tabular}{|c|c|c|c|c|c|c|c|}
\hline Sample & \multicolumn{2}{|c|}{$\begin{array}{l}\text { Depth } \\
\text { (feet) }\end{array}$} & $\begin{array}{l}\mathrm{Mn}(\operatorname{carb}) \\
\mathrm{mg} / \mathrm{kg}\end{array}$ & $\begin{array}{l}\mathrm{Mn}(\mathrm{MnO}) \\
\mathrm{mg} / \mathrm{kg}\end{array}$ & $\begin{array}{l}\text { Mn }(\operatorname{amfe}) \\
\mathrm{mg} / \mathrm{kg}\end{array}$ & $\begin{array}{l}\mathrm{Mn}(\text { cryfe) } \\
\mathrm{mg} / \mathrm{kg}\end{array}$ & $\begin{array}{l}\mathrm{Mn} \text { (res) } \\
\mathrm{mg} / \mathrm{kg}\end{array}$ \\
\hline$x 1-107 b 1$ & \multicolumn{2}{|l|}{16} & 22 & 292 & 83 & 59 & 800 \\
\hline$\times 1-107 b 2$ & \multicolumn{2}{|l|}{17} & 27 & 393 & 210 & 163 & 449 \\
\hline$\times 1-107 b 3$ & \multicolumn{2}{|c|}{17.5} & 88 & 18,700 & 6,300 & 67 & 430 \\
\hline$x 1-107 b 4$ & \multicolumn{2}{|c|}{18} & 93. & 16.5 & 46 & 30 & 549 \\
\hline x1-107b5 & 19 & -24 & 84 & 6.6 & 53 & 46 & 620 \\
\hline$x 1-107 b 6$ & 38 & -39 & 99 & 5.7 & 32 & 138 & 459 \\
\hline$x 1-107 b 7$ & 46 & -47 & 68 & 2.8 & 28 & 22 & 1,079 \\
\hline$x 1-107 b 8$ & 56 & -57 & 58 & 5.6 & 28 & 128 & 722 \\
\hline$x 1-107 b 9$ & 71 & -72 & 57 & 7.1 & 21 & 88 & 904 \\
\hline$x 5-505 b 1$ & \multicolumn{2}{|c|}{$0.7-1.1$} & 13 & 45,600 & 1,390 & 89 & 760 \\
\hline$x 5-505 b 2$ & \multicolumn{2}{|c|}{$1.1-2$} & 26 & 1,120 & 118 & 64 & 1,470 \\
\hline$x 5-505 b 3$ & 9 & -10 & 57 & 485 & 86 & 77 & 730 \\
\hline$x 5-505 b 4$ & 10 & -11.5 & 163 & 3,200 & 548 & 104 & 490 \\
\hline$x 5-505 b 5$ & 18 & -21.5 & 84 & 173 & 59 & 80 & 1,360 \\
\hline$x 5-505 b 6$ & 23 & -24.8 & 89 & 155 & 73 & 82 & 1,200 \\
\hline$x 5-505 b 7$ & 58 & -60 & 183 & 868 & 243 & 69 & 690 \\
\hline$x 5-505 b 8$ & 60 & -61.5 & 153 & 740 & 164 & 89 & 650 \\
\hline $\mathrm{x} 5-505 \mathrm{bg}$ & 64 & -65 & 15 & 228 & 119 & 71 & 370 \\
\hline$x 5-505 b 10$ & 71 & -73 & 22 & 215 & 97 & 100 & 210 \\
\hline$x 5-506 b 1$ & & $8-1.2$ & 4 & 26,000 & 1,090 & 53 & 1,010 \\
\hline$x 5-506 b 2$ & 10. & $5-11.5$ & 112 & 125 & 91 & 43 & 730 \\
\hline$x 5-506 b 3$ & 19 & -22 & 107 & 389 & 134 & 134 & 578 \\
\hline$x 5-506$ & 23 & -24.8 & 101 & 253 & 79 & 116 & 1,230 \\
\hline $451 b 1$ & 10. & & 45 & 1,531 & 275 & 59 & 1,060 \\
\hline $451 \mathrm{~b} 2$ & 11 & & 33 & 588 & 168 & 120 & 890 \\
\hline $451 \mathrm{b3}$ & 12 & & 98 & 730 & 240 & 79 & 910 \\
\hline $451 \mathrm{~b} 4$ & 13 & & 66 & 203 & 68 & 29 & 560 \\
\hline 451 & 21 & & 232 & 304 & 97 & 136 & 570 \\
\hline $451 b 5$ & 30 & -31 & 192 & 160 & 74 & 102 & 1,360 \\
\hline $451 b 6$ & 32 & & 225 & 516 & 168 & 57 & 890 \\
\hline $451 b 7$ & 36 & & 324 & 142 & 121 & 108 & 1,340 \\
\hline $451 b 8$ & 37. & $5-38$ & 228 & 152 & 107 & 57 & 1,780 \\
\hline $451 b 9$ & 42. & & 159 & 227 & 97 & 140 & 1,122 \\
\hline 451 & 51. & $8-52.3$ & 235 & 154 & 89 & 82 & 720 \\
\hline $451 \mathrm{~b} 10$ & 57 & & 174 & 209 & 76 & 85 & 1,294 \\
\hline $451 b 11$ & 70 & -73 & 174 & 14 & 41 & 100 & 1,520 \\
\hline $451 b 12$ & 76. & $5-70$ & 224 & 17 & 39 & 117 & 1,000 \\
\hline $452 \mathrm{bl}$ & 3 & -6 & 10 & 191 & 64 & 88 & 1,804 \\
\hline $452 \mathrm{~b} 2$ & 6. & $5-7$ & 9.4 & 259 & 57 & 74 & 2,140 \\
\hline $452 \mathrm{~b} 3$ & 11 & -12 & 100 & $1,440.2$ & 385 & 125 & 540 \\
\hline
\end{tabular}


Table 3. Analytical results for partial sequential extractions of Pinal Creek aquifer material--(Continued)

\begin{tabular}{|c|c|c|c|c|c|c|c|}
\hline Sample & \multicolumn{2}{|c|}{$\begin{array}{l}\text { Depth } \\
\text { (feet) }\end{array}$} & $\begin{array}{l}\mathrm{Mn} \text { (carb) } \\
\mathrm{mg} / \mathrm{kg}\end{array}$ & $\begin{array}{l}\mathrm{Mn}(\mathrm{MnO}) \\
\mathrm{mg} / \mathrm{kg}\end{array}$ & $\begin{array}{l}\mathrm{Mn}(\operatorname{amfe}) \\
\mathrm{mg} / \mathrm{kg}\end{array}$ & $\begin{array}{l}M n(\operatorname{cryfe}) \\
\mathrm{mg} / \mathrm{kg}\end{array}$ & $\begin{array}{l}\mathrm{Mn} \text { (res) } \\
\mathrm{mg} / \mathrm{kg}\end{array}$ \\
\hline 452 & \multicolumn{2}{|c|}{$14.8-15$} & 250 & 296 & 216 & 79 & 200 \\
\hline $452 \mathrm{~b} 4$ & 18 & -19 & 159 & 376 & 129 & 112 & 920 \\
\hline $452 b 5$ & 19 & -21 & 180 & 275 & 113 & 97 & 1,190 \\
\hline $452 b 6$ & 23 & -23 & 193 & 334 & 121 & 140 & 2,022 \\
\hline 010b1 & 5 & -10 & 57 & 108 & 57 & 38 & 978 \\
\hline $010 \mathrm{~b} 2$ & 15 & -20 & 105 & 31 & 31 & 25 & 1,000 \\
\hline $010 \mathrm{b3}$ & 23 & -25 & 92 & 70 & 81 & 180 & 380 \\
\hline $010 b 4$ & 29 & -30 & 77 & 85 & 60 & 40 & 965 \\
\hline $010 b 5$ & 37 & -38 & 39 & 114 & 69 & 169 & 560 \\
\hline $010 \mathrm{~b} 6$ & 45 & -46 & 64 & 123 & 74 & 168 & 500 \\
\hline $010 \mathrm{~b} 7$ & 50 & -52 & 136 & 31 & 58 & 141 & 440 \\
\hline $010 \mathrm{~b} 8$ & 57. & $5-60$ & 56 & 48 & 81 & 169 & 380 \\
\hline 010b9 & 60 & -63 & 20 & 24 & 57 & 100 & 220 \\
\hline 010b10 & 63 & -67 & 25 & 56 & 118 & 191 & 280 \\
\hline 010b11 & 70 & -75 & 26 & 107 & 64 & 197 & 170 \\
\hline 010b12 & 86 & -90 & 40 & 91 & 69 & 185 & 360 \\
\hline
\end{tabular}


Table 3. Analytical results for partial sequential extractions of Pinal Creek aquifer material--(Continued)

\begin{tabular}{|c|c|c|c|c|c|c|c|}
\hline Sample & \multicolumn{2}{|c|}{$\begin{array}{l}\text { Depth } \\
\text { (feet) }\end{array}$} & $\begin{array}{l}\mathrm{Fe}(\mathrm{carb}) \\
\mathrm{mg} / \mathrm{kg}\end{array}$ & $\begin{array}{l}\mathrm{Fe}(\mathrm{MnO}) \\
\mathrm{mg} / \mathrm{kg}\end{array}$ & $\begin{array}{l}\mathrm{Fe}(\operatorname{amfe}) \\
\mathrm{mg} / \mathrm{kg}\end{array}$ & $\begin{array}{l}\mathrm{Fe}(\text { cryfe }) \\
\mathrm{mg} / \mathrm{kg}\end{array}$ & $\begin{array}{l}\mathrm{Fe} \text { (res) } \\
\mathrm{mg} / \mathrm{kg}\end{array}$ \\
\hline $1-107 \mathrm{bl}$ & \multicolumn{2}{|l|}{16} & 111 & 110 & 5,200 & 13,900 & 60,400 \\
\hline$x 1-107 b 2$ & \multicolumn{2}{|l|}{17} & 27 & 37 & 2,470 & 24,157 & 19,662 \\
\hline$x 1-107 b 3$ & \multicolumn{2}{|c|}{17.5} & 187 & 600 & 6,300 & 10,000 & 33,300 \\
\hline$x 1-107 b 4$ & \multicolumn{2}{|c|}{18} & 269 & 85 & 2,939 & 4,505 & 42,857 \\
\hline$x 1-107 b 5$ & 19 & -24 & 184 & 98 & 4,900 & 13,200 & 40,800 \\
\hline$x 1-107 b 6$ & 38 & -39 & 275 & 83 & 1,520 & 21,300 & 34,482 \\
\hline$x 1-107 b 7$ & 46 & -47 & 494 & 79 & 1,676 & 4,261 & 72,727 \\
\hline$x 1-107 b 8$ & 56 & -57 & 513 & 72 & 2,190 & 26,700 & 55,555 \\
\hline x1-107b9 & 71 & -72 & 950 & 98 & 1,260 & 19.500 & 70,476 \\
\hline$x 5-505 b 1$ & \multicolumn{2}{|c|}{$0.7-1.1$} & 50 & 411 & 5,400 & 11,100 & 39,900 \\
\hline$x 5-505 b 2$ & \multicolumn{2}{|c|}{$1.1-2$} & 270 & 280 & 5,360 & 12,800 & 66,700 \\
\hline$x 5-505 b 3$ & 9 & -10 & 210 & 240 & 3,750 & 16,700 & 39,300 \\
\hline$\times 5-505 b 4$ & 10 & -11.5 & 77 & 244 & 4,300 & 13,500 & 26,800 \\
\hline$x 5-505 b 5$ & 18 & -21.5 & 95 & 123 & 3,100 & 15,400 & 53,100 \\
\hline$x 5-505 b 6$ & 23 & -24.8 & 97 & 158 & 5,200 & 19,600 & 47,500 \\
\hline$x 5-505 b 7$ & 58 & -60 & 220 & 277 & 4,270 & 10,300 & 40,300 \\
\hline$x 5-505 b 8$ & 60 & -61.5 & 150 & 247 & 3,870 & 14,200 & 46,900 \\
\hline$x 5-505 b 9$ & 64 & -65 & 49 & 61 & 980 & 7,600 & 29,900 \\
\hline$x 5-505 b 10$ & 71 & -73 & 50 & 35 & 870 & 13,900 & 13,900 \\
\hline$x 5-506 b 1$ & & $8-1.2$ & 20 & 328 & 5,100 & 12,600 & 73,700 \\
\hline$x 5-506 b 2$ & 10. & $5-11.5$ & 73 & 91 & 4,900 & 9,100 & 49,400 \\
\hline$x 5-506 b 3$ & 19 & -22 & 55 & 53 & 2,580 & 20,500 & 34,736 \\
\hline$x 5-506$ & 23 & -24.8 & 87 & 181 & 1,800 & 17,750 & 47,100 \\
\hline $451 \mathrm{bl}$ & 10. & & 50 & 94 & 3,100 & 12,500 & 75,000 \\
\hline $451 \mathrm{~b} 2$ & 11 & & 33 & 35 & 2,090 & 16,500 & 59,500 \\
\hline $451 b 3$ & 12 & & 49 & 91 & 3,400 & 12,200 & 59,800 \\
\hline $451 b 4$ & 13 & & 140 & 88 & 3,200 & 8,800 & 52,900 \\
\hline 451 & 21 & & 52 & 54 & 1,900 & 18,800 & 40,300 \\
\hline $451 b 5$ & 30 & -31 & 96 & 55 & 2,190 & 15,400 & 93,800 \\
\hline $451 b 6$ & 32 & & 76 & 158 & 4,430 & 12,900 & 57,600 \\
\hline $451 \mathrm{~b} 7$ & 36 & & 41 & 43 & 4,030 & 24,700 & 82,300 \\
\hline $451 \mathrm{~b} 8$ & 37. & $5-38$ & 95 & 89 & 4,500 & 17,900 & 108,000 \\
\hline $451 b 9$ & 42. & & 41 & 51 & 2,500 & 21,400 & 64,285 \\
\hline 451 & 51. & $8-52.3$ & 110 & 171 & 5,500 & 17,100 & 43,800 \\
\hline $451 \mathrm{~b} 10$ & 57 & & 26 & 47 & 2,000 & 14,700 & 89.411 \\
\hline $451 \mathrm{bl1}$ & 70 & -73 & 260 & 81 & 2,720 & 19,600 & 125,000 \\
\hline $451 b 12$ & 76. & $5-70$ & 240 & 64 & 1,360 & 27,200 & 68,888 \\
\hline $452 \mathrm{bl}$ & & -6 & 23 & 28 & 695 & 17,500 & 92,783 \\
\hline $452 \mathrm{~b} 2$ & & $5-7$ & 17 & 24 & 1,400 & 12,500 & 149,000 \\
\hline
\end{tabular}


Table 3. Analytical results for partial sequential extractions of Pinal Creek aquifer material--(Continued)

\begin{tabular}{|c|c|c|c|c|c|c|c|}
\hline \multirow{2}{*}{$\begin{array}{l}\text { Sample } \\
452 \mathrm{~b} 3\end{array}$} & \multicolumn{2}{|c|}{$\begin{array}{l}\text { Depth } \\
\text { (feet) }\end{array}$} & \multirow{2}{*}{$\frac{\begin{array}{l}\mathrm{Fe}(\mathrm{carb}) \\
\mathrm{mg} / \mathrm{kg}\end{array}}{20}$} & \multirow{2}{*}{$\begin{aligned} \mathrm{Fe}(\mathrm{MnO}) \\
\mathrm{mg} / \mathrm{kg}\end{aligned}$} & \multirow{2}{*}{$\begin{array}{c}\begin{array}{l}\mathrm{Fe}(\operatorname{amfe}) \\
\mathrm{mg} / \mathrm{kg}\end{array} \\
2,170\end{array}$} & \multirow{2}{*}{$\begin{array}{l}\text { Fe (cryfe) } \\
\mathrm{mg} / \mathrm{kg}\end{array}$} & \multirow{2}{*}{$\begin{array}{l}\mathrm{Fe} \text { (res) } \\
\mathrm{mg} / \mathrm{kg}\end{array}$} \\
\hline & 11 & -12 & & & & & \\
\hline 452 & 14. & $8-15$ & 13 & 19 & 2,310 & 25,500 & 23,100 \\
\hline $452 \mathrm{~b} 4$ & 18 & -19 & 39 & 51 & 2,060 & 14,100 & 65,000 \\
\hline $452 \mathrm{~b} 5$ & 19 & -21 & 30 & 47 & 2,410 & 22,500 & 85,600 \\
\hline $452 \mathrm{~b} 6$ & 23 & -23 & 36 & 42 & 730 & 34,100 & 101,123 \\
\hline 010b1 & 5 & -10 & 76 & 87 & 1,521 & 6,250 & 26,087 \\
\hline $010 \mathrm{~b} 2$ & 15 & -20 & 308 & 670 & 1,700 & 4,170 & 83,300 \\
\hline $010 b 3$ & 23 & -25 & 217 & 545 & 4,270 & 29,600 & 22,600 \\
\hline 010b4 & 29 & -30 & 80 & 91 & 2,100 & 6,600 & 85,200 \\
\hline $010 b 5$ & 37 & -38 & 69 & 92 & 1,690 & 36,600 & 37,200 \\
\hline $010 b 6$ & 45 & -46 & 132 & 220 & 2,380 & 31,100 & 34,400 \\
\hline 010b7 & 50 & -52 & 460 & 670 & 3,030 & 14,400 & 51,700 \\
\hline $010 b 8$ & 57. & $5-60$ & 133 & 126 & 4,060 & 28,000 & 18,300 \\
\hline 010b9 & 60 & -63 & 80 & 64 & 1,520 & 10,900 & 18,500 \\
\hline 010b10 & 63 & -67 & 47 & 17 & 101 & 21,500 & 16,700 \\
\hline $010 b 11$ & 70 & -75 & 37 & 81 & 523 & 20,300 & 14,500 \\
\hline 010b12 & 86 & -90 & 81 & 127 & 101 & 23,200 & 18,800 \\
\hline
\end{tabular}


Table 3. Analytical results for partial sequential extractions of Pinal Creek aquifer material--(Continued)

\begin{tabular}{|c|c|c|c|c|c|c|}
\hline Sample & \multicolumn{2}{|c|}{$\begin{array}{l}\text { Depth } \\
\text { (feet) }\end{array}$} & $\begin{array}{c}\mathrm{SO}_{4}(\mathrm{carb}) \\
\mathrm{mg} / \mathrm{kg}\end{array}$ & $\begin{array}{c}\mathrm{SO}_{4}(\mathrm{MnO}) \\
\mathrm{mg} / \mathrm{kg}\end{array}$ & $\begin{array}{c}\mathrm{SO}_{4} \text { (amfe) } \\
\mathrm{mg} / \mathrm{kg}\end{array}$ & $\begin{array}{c}\mathrm{SO}_{4} \text { (tot) } \\
\mathrm{mg} / \mathrm{kg}\end{array}$ \\
\hline$x 1-107 b 1$ & \multicolumn{2}{|l|}{16} & 917 & 101 & 45 & $<1,500$ \\
\hline$x 1-107 b 2$ & \multicolumn{2}{|l|}{17} & 1,690 & 147 & 75 & 1,500 \\
\hline$x 1-107 b 3$ & \multicolumn{2}{|c|}{17.5} & 1,770 & 117 & 234 & 1,800 \\
\hline$x 1-107 b 4$ & \multicolumn{2}{|c|}{18} & 1,169 & 150 & 78 & 1,500 \\
\hline$x 1-107 b 5$ & 19 & -24 & 812 & 74 & 360 & $<1,500$ \\
\hline$x 1-107 b 6$ & 38 & -39 & 1,611 & 120 & 105 & 2,100 \\
\hline$x 1-107 b 7$ & 46 & -47 & 2,372 & 150 & 92 & 2,400 \\
\hline$x 1-107 b 8$ & 56 & -57 & 1,598 & 136 & 105 & 3,000 \\
\hline$x 1-107 b 9$ & 71 & -72 & 1,1000 & 406 & 144 & 13,800 \\
\hline$x 5-505 b 1$ & \multicolumn{2}{|c|}{$0.7-1.1$} & 792 & 101 & 245 & 1,500 \\
\hline$x 5-505 b 2$ & \multicolumn{2}{|c|}{$1.1-2$} & 419 & 43 & 223 & $<1,500$ \\
\hline$x 5-505 b 3$ & 9 & -10 & 1,562 & 84 & 50 & 1,800 \\
\hline$x 5-505 b 4$ & 10 & -11.5 & 1,991 & 117 & 159 & 2,400 \\
\hline$x 5-505 b 5$ & 18 & -21.5 & 1,185 & 63 & 237 & 1,800 \\
\hline$x 5-505 b 6$ & 23 & -24.8 & 1,586 & 956 & 78 & 2,100 \\
\hline$x 5-505 b 7$ & 58 & -60 & 1,566 & 177 & 67 & 1,800 \\
\hline$x 5-505 b 8$ & 60 & -61.5 & 1,313 & 131 & 55 & 1,500 \\
\hline$x 5-505 b 9$ & 64 & -65 & 758 & 138 & 40 & $<1,500$ \\
\hline$x 5-505 b 10$ & 71 & -73 & 834 & 150 & 51 & $<1,500$ \\
\hline$x 5-506 b 1$ & & $8-1.2$ & 419 & 59 & 278 & $<1,500$ \\
\hline$x 5-506 b 2$ & 10. & $5-11.5$ & 3,199 & 85 & 41 & 3,000 \\
\hline$x 5-506 b 3$ & 19 & -22 & $<50$ & 76 & 61 & 1,500 \\
\hline$x 5-506$ & 23 & -24.8 & 2,000 & 188 & 65 & 1,800 \\
\hline $451 b 1$ & 10. & & 522 & 81 & $<50$ & $<1,500$ \\
\hline $451 \mathrm{~b} 2$ & 11 & & 832 & 131 & 59 & $<1,500$ \\
\hline $451 b 3$ & 12 & & 4,746 & 112 & 100 & 5,700 \\
\hline $451 b 4$ & 13 & , & 4,900 & 120 & 100 & $<1,500$ \\
\hline 451 & 21 & & 2,024 & 122 & 80 & 1,500 \\
\hline $451 b 5$ & 30 & -31 & 6,713 & 266 & 48 & 38,900 \\
\hline $451 \mathrm{~b} 6$ & 32 & & 1,911 & 68 & 63 & 1,800 \\
\hline $451 \mathrm{~b} 7$ & 36 & & 4,590 & 164 & 72 & $<1,500$ \\
\hline $451 b 8$ & 37. & $5-38$ & 1,660 & 106 & $<50$ & 1,800 \\
\hline $451 b 9$ & 42. & & 718 & 76 & $<50$ & $<1,500$ \\
\hline 451 & 51. & $8-52.3$ & 2,968 & 92 & 75 & 1,500 \\
\hline $451 \mathrm{~b} 10$ & 57 & & 1,437 & 235 & 57 & 1,500 \\
\hline $451 \mathrm{~b} 11$ & 70 & -73 & 4,589 & 218 & 83 & 5,100 \\
\hline $451 b 12$ & 76. & $5-70$ & 2,582 & 123 & 84 & 2,700 \\
\hline $452 \mathrm{bl}$ & 3 & -6 & 360 & 38 & $<50$ & $<1,500$ \\
\hline $452 \mathrm{~b} 2$ & & $5-7$ & $<40$ & 114 & $<50$ & $<1,500$ \\
\hline
\end{tabular}


Table 3. Analytical results for partial sequential extractions of Pinal Creek aquifer material--(Continued)

\begin{tabular}{|c|c|c|c|c|c|c|}
\hline \multirow{2}{*}{$\begin{array}{l}\text { Sample } \\
452 \mathrm{b3}\end{array}$} & \multicolumn{2}{|c|}{$\begin{array}{l}\text { Depth } \\
\text { (feet) }\end{array}$} & \multirow{2}{*}{$\begin{array}{c}\mathrm{so}_{4}(\mathrm{carb}) \\
\mathrm{mg} / \mathrm{kg}\end{array}$} & \multirow{2}{*}{$\frac{\begin{array}{c}\mathrm{SO}_{4}(\mathrm{MnO}) \\
\mathrm{mg} / \mathrm{kg}\end{array}}{166}$} & \multirow{2}{*}{$\begin{array}{c}\mathrm{So}_{4}(\operatorname{amfe}) \\
\mathrm{mg} / \mathrm{kg}\end{array}$} & \multirow{2}{*}{$\begin{array}{c}\mathrm{so}_{4} \text { (tot) } \\
\mathrm{mg} / \mathrm{kg} \\
2,100\end{array}$} \\
\hline & 11 & -12 & & & & \\
\hline 452 & 14. & $8-15$ & 707 & 168 & 43.0 & $<1,500$ \\
\hline $452 b 4$ & 18 & -19 & 1,940 & 6,274 & 72.0 & 3,000 \\
\hline $452 \mathrm{~b} 5$ & 19 & -21 & 3,147 & 228 & 70.0 & 3,000 \\
\hline $452 \mathrm{~b} 6$ & 23 & -23 & 3,058 & 213 & 84.0 & $2, ; 00$ \\
\hline 010b1 & 5 & -10 & 276 & $<50$ & $<50$ & $<1,500$ \\
\hline 010b2 & 15 & -20 & 213 & $<50$ & $<50$ & $<1,500$ \\
\hline $010 b 3$ & 23 & -25 & 338 & $<50$ & $<50$ & $<1,500$ \\
\hline $010 \mathrm{~b} 4$ & 29 & -30 & 188 & $<50$ & $<50$ & $<1,500$ \\
\hline $010 b 5$ & 37 & -38 & 293 & $<50$ & $<50$ & $<1,500$ \\
\hline $010 \mathrm{~b} 6$ & 45 & -46 & 341 & $<50$ & $<50$ & $<1,500$ \\
\hline $010 b 7$ & 50 & -52 & 202 & 288 & $<50$ & $<1,500$ \\
\hline $010 b 8$ & 57. & $5-60$ & 307 & 105 & $<50$ & $<1,500$ \\
\hline $010 b 9$ & 60 & -63 & 193 & $<50$ & $<50$ & $<1,500$ \\
\hline 010b10 & 63 & -67 & 467 & $<50$ & $<50$ & $<1,500$ \\
\hline 010b11 & 70 & -75 & $<40$ & 120 & $<50$ & $<1,500$ \\
\hline $010 b 12$ & 86 & -90 & 489 & 140 & $<50$ & $<1,500$ \\
\hline
\end{tabular}


Table 3. Analytical results for partial sequential extractions of Pinal Creek aquifer material--(Continued)

\begin{tabular}{|c|c|c|c|c|c|}
\hline Sample & $\begin{array}{l}\text { Depth } \\
\text { (feet) }\end{array}$ & $\begin{array}{l}\mathrm{Ca}(\mathrm{carb}) \\
\mathrm{mg} / \mathrm{kg}\end{array}$ & $\begin{array}{l}\mathrm{Ca}(\mathrm{MnO}) \\
\mathrm{mg} / \mathrm{kg}\end{array}$ & $\begin{array}{l}\mathrm{Ca} \text { (amfe) } \\
\mathrm{mg} / \mathrm{kg}\end{array}$ & $\begin{array}{l}\text { Ca (cryfe) } \\
\mathrm{mg} / \mathrm{kg}\end{array}$ \\
\hline$x 1-107 b 1$ & 16 & 1,875 & 260 & 1,996 & 173 \\
\hline$\times 1-107 b 2$ & 17 & 3,988 & 84 & 1,825 & 81 \\
\hline$x 1-107 b 3$ & 17.5 & 2,667 & 107 & 1,666 & 10 \\
\hline$x 1-107 b 4$ & 18 & 1,593 & 38 & 1.373 & 27 \\
\hline$\times 1-107 b 5$ & $19-24$ & 1,381 & 85 & 1,562 & 10 \\
\hline$x 1-107 b 6$ & $\begin{array}{ll}38 & -39\end{array}$ & 2,068 & 34 & 1,795 & 49 \\
\hline$x 1-107 b 7$ & $46-47$ & 1,420 & 25 & - & 37 \\
\hline$x 1-107 b 8$ & $\begin{array}{ll}56 & -57\end{array}$ & 1,000 & 11 & 1,944 & 42 \\
\hline$x 1-107 b 9$ & $71-72$ & 4,666 & 81 & 1,726 & 28 \\
\hline$x 5-505 b 1$ & $0.7-1.1$ & 4,810 & 1,424 & 2,056 & 22 \\
\hline$\times 5-505 b 2$ & $1.1-2$ & 1,089 & 320 & 2,083 & 22 \\
\hline$x 5-505 b 3$ & $9-10$ & 2,023 & 357 & 1,711 & 27 \\
\hline$x 5-505 b 4$ & $10-11.5$ & 2,804 & 262 & 1,753 & 40 \\
\hline$\times 5-505 b 5$ & $\begin{array}{ll}18 & -21.5\end{array}$ & 1,419 & 40 & 2,160 & 28 \\
\hline$x 5-505 b 6$ & $23-24.8$ & 2,848 & 411 & 3,401 & 41 \\
\hline$\times 5-505 b 7$ & $\begin{array}{ll}58 & -60\end{array}$ & 3,670 & 322 & 2,083 & 31 \\
\hline$x 5-505 b 8$ & $60-61.5$ & 3,518 & 324 & 1,697 & 43 \\
\hline$\times 5-505 b 9$ & $64-65$ & 20,120 & 396 & 1,829 & 82 \\
\hline$x 5-505 b 10$ & $\begin{array}{ll}71 & -73\end{array}$ & 33,300 & - & 1,475 & 24 \\
\hline$x 5-506 b 1$ & $0.8-1.2$ & 4,343 & 820 & 3,724 & 10 \\
\hline$x 5-506 b 2$ & $10.5-11.5$ & 4,268 & 189 & 2,286 & 18 \\
\hline$x 5-506 b 3$ & $19-22$ & 2,105 & 60 & 2,105 & 115 \\
\hline$x 5-506$ & $23-24.8$ & 2,863 & 1,449 & 2,264 & 40 \\
\hline $451 \mathrm{bl}$ & 10.5 & 2,062 & 128 & 3,203 & 19 \\
\hline $451 \mathrm{~b} 2$ & 11 & 2,215 & 85 & 2,848 & 19 \\
\hline $451 \mathrm{~b} 3$ & 12 & - & 304 & 2,667 & 24 \\
\hline$\angle 51 \mathrm{~b} 4$ & 13 & 1,235 & 103 & 1,764 & 8.8 \\
\hline 451 & 21 & 4,659 & 3,053 & 14,204 & 144 \\
\hline $451 b 5$ & $30-31$ & 4,135 & 99 & 2,237 & 52 \\
\hline $451 b 6$ & 32 & 2,215 & 221 & 1,977 & 16 \\
\hline $451 b 7$ & 36 & 2,903 & 65 & 3,696 & 72 \\
\hline $451 b 8$ & $37.5-38$ & 1,753 & 372 & 2,380 & 228 \\
\hline $451 b 9$ & 42.5 & 1,428 & 33 & 2,487 & 112 \\
\hline 451 & $51.8-52.3$ & 1,683 & 260 & 86 & 31 \\
\hline $451 b 10$ & 57 & 1,705 & 26 & 3,014 & 38 \\
\hline $451 b 11$ & $70-73$ & 2,717 & 24 & 2,649 & 57 \\
\hline $451 b 12$ & $76.5-70$ & 2,666 & 50 & 2,083 & 92 \\
\hline $452 \mathrm{bl}$ & $3-6$ & 3,505 & 93 & 3,092 & 52 \\
\hline $452 \mathrm{~b} 2$ & $6.5-7$ & 2,083 & 104 & 3,422 & 39 \\
\hline $452 \mathrm{~b} 3$ & $11-12$ & 3,315 & 141 & 2,241 & 35 \\
\hline
\end{tabular}


Table 3. Analytical results for partial sequential extractions of Pinal Creek aquifer material--(Continued)

\begin{tabular}{llllll}
\hline Sample & $\begin{array}{l}\text { Depth } \\
\text { (feet) }\end{array}$ & $\begin{array}{l}\mathrm{Ca}(\mathrm{carb}) \\
\mathrm{mg} / \mathrm{kg}\end{array}$ & $\begin{array}{l}\mathrm{Ca}(\mathrm{MnO}) \\
\mathrm{mg} / \mathrm{kg}\end{array}$ & $\begin{array}{l}\mathrm{Ca}(\mathrm{mfe}) \\
\mathrm{mg} / \mathrm{kg}\end{array}$ & $\begin{array}{l}\mathrm{Ca}(\mathrm{cryfe}) \\
\mathrm{mg} / \mathrm{kg}\end{array}$ \\
\hline
\end{tabular}

\begin{tabular}{|c|c|c|c|c|c|c|}
\hline 452 & \multicolumn{2}{|c|}{$14.8-15$} & 4,807 & 103 & 841 & 84 \\
\hline $452 \mathrm{~b} 4$ & 18 & -19 & 1,941 & 44 & 2,730 & 63 \\
\hline $452 \mathrm{~b} 5$ & 19 & -21 & 3,125 & 69 & 3,359 & 44 \\
\hline $452 \mathrm{~b} 6$ & 23 & -23 & 2,865 & 76 & 2,668 & 101 \\
\hline $010 \mathrm{bl}$ & 5 & -10 & 5,108 & 111 & 2,309 & 0 \\
\hline $010 \mathrm{~b} 2$ & 15 & -20 & 1,500 & 58 & 1,805 & 17 \\
\hline $010 b 3$ & 23 & -25 & 1,774 & 62 & 1,680 & 86 \\
\hline $010 b 4$ & 29 & -30 & 2,329 & 71 & 2,982 & 45 \\
\hline $010 b 5$ & 37 & -38 & 2,388 & 56 & 2,152 & 7 \\
\hline $010 b_{6}$ & 45 & -46 & 1,117 & 56 & 1,764 & 7 \\
\hline $010 \mathrm{~b} 7$ & 50 & -52 & 1,333 & 50 & 1,458 & 3 \\
\hline $010 \mathrm{~b} 8$ & 57. & $5-60$ & 1,989 & 48 & 2,217 & \\
\hline 010b9 & 60 & -63 & 2,336 & 11 & 1,834 & \\
\hline 010b10 & 63 & -67 & 2,083 & 180 & 1,302 & 69 \\
\hline $010 b 11$ & 70 & -75 & 48,837 & 1,017 & 1,671 & 11 \\
\hline $010 \mathrm{~b} 12$ & 86 & -90 & 27,536 & 362 & 1,811 & 90 \\
\hline
\end{tabular}


Table 3. Analytical results for partial sequential extractions of Pinal Creek aquifer material--(Continued)

\begin{tabular}{|c|c|c|c|c|c|}
\hline Sample & $\begin{array}{l}\text { Depth } \\
\text { (feet) }\end{array}$ & $\begin{array}{l}\mathrm{Al} \text { (carb) } \\
\mathrm{mg} / \mathrm{kg}\end{array}$ & $\begin{array}{l}\mathrm{Al}(\mathrm{MnO}) \\
\mathrm{mg} / \mathrm{kg}\end{array}$ & $\begin{array}{l}\text { Al (amfe) } \\
\mathrm{mg} / \mathrm{kg}\end{array}$ & $\begin{array}{l}\text { Al (cryfe) } \\
\mathrm{mg} / \mathrm{kg}\end{array}$ \\
\hline$x 1-107 b 1$ & 16 & 25 & 76 & 1,597 & 2,986 \\
\hline$x 1-107 b 2$ & 17 & 40 & 53 & - & 9,691 \\
\hline$x 1-107 b 3$ & 17.5 & 88 & 111 & 2,233 & 2,300 \\
\hline$x 1-107 b 4$ & 18 & 44 & 36 & 906 & 1,318 \\
\hline$x 1-107 b 5$ & $19-24$ & 63 & 76 & 1,348 & 2,368 \\
\hline$x 1-107 b 6$ & $\begin{array}{ll}38 & -39\end{array}$ & 78 & 32 & 1,005 & 6,178 \\
\hline$x 1-107 b 7$ & $\begin{array}{ll}46 & -47\end{array}$ & 261 & 23 & 1,193 & 1,250 \\
\hline$x 1-10768$ & $\begin{array}{ll}56 & -57\end{array}$ & 200 & 55 & 1,055 & 5,194 \\
\hline$\times 1-107 b 9$ & $\begin{array}{ll}71 & -72\end{array}$ & 304 & 28 & 1,023 & 36,666 \\
\hline$x 5-505 b 1$ & $0.7-1.1$ & 58 & 92 & 1,234 & 2,563 \\
\hline$x 5-505 b 2$ & $1.1-2$ & 49 & 86 & 769 & 2,147 \\
\hline$x 5-505 b 3$ & $9-10$ & 33 & 116 & 1,220 & 3,273 \\
\hline$x 5-505 b 4$ & $10-11.5$ & 44 & 76 & 1,768 & 4,116 \\
\hline$x 5-505 b 5$ & $\begin{array}{ll}18 & -21.5\end{array}$ & 22 & 123 & 1,080 & 3,055 \\
\hline$x 5-505 b 6$ & $\begin{array}{ll}23 & -24.8\end{array}$ & 38 & 126 & 1,898 & 4,556 \\
\hline$x 5-505 b 7$ & $58-60$ & 61 & 139 & 1,527 & 3,576 \\
\hline$x 5-505 b 8$ & $60-61.5$ & 31 & 111 & 1,543 & 4,367 \\
\hline$x 5-505 b 9$ & $64-65$ & 83 & 112 & 1,128 & 3,506 \\
\hline$x 5-505 b 20$ & $\begin{array}{ll}71 & -73\end{array}$ & 81 & - & 1,076 & 3,368 \\
\hline$x 5-506 b 1$ & $0.8-1.2$ & 52 & 38 & 1,338 & 1,464 \\
\hline$x 5-506 b 2$ & $10.5-11.5$ & 56 & 119 & 1,890 & 2,286 \\
\hline$x 5-506 b 3$ & $19-22$ & 48 & 26 & 1,157 & 5,789 \\
\hline$x 5-506$ & $\begin{array}{ll}23 & -24.8\end{array}$ & 50 & 192 & 1,340 & 4,710 \\
\hline $452 \mathrm{bl}$ & 10.5 & 35 & 153 & 1,438 & 2,125 \\
\hline $451 \mathrm{~b} 2$ & 11 & 38 & 25 & 1,170 & 5,759 \\
\hline $451 \mathrm{b3}$ & 12 & 49 & 131 & 2,073 & 3,536 \\
\hline $451 \mathrm{~b} 4$ & 13 & 49 & 65 & 970 & 1,529 \\
\hline 451 & 21 & 45 & 48 & 937 & 5,227 \\
\hline $451 b 5$ & $30-31$ & 44 & 25 & 1,049 & 4,290 \\
\hline $451 b 6$ & 32 & 68 & 107 & 1,709 & 2,183 \\
\hline $451 b 7$ & 36 & 47 & 43 & 1,559 & 5,000 \\
\hline $451 b 8$ & $37.5-38$ & 52 & 98 & 1,428 & 1,994 \\
\hline 45169 & 42.5 & 43 & 64 & 1,020 & 5,357 \\
\hline 451 & $51.8-52.3$ & 71 & 123 & 1,815 & 3,493 \\
\hline $451 b 10$ & 57 & 35 & 74 & 911 & 2,970 \\
\hline $451 \mathrm{~b} 11$ & $70-73$ & 76 & 100 & 1,250 & 4,076 \\
\hline $451 b 12$ & $76.5-70$ & 87 & 33 & 1,222 & 6,028 \\
\hline $452 \mathrm{bl}$ & $3-6$ & 33 & 15 & 798 & 3,222 \\
\hline $452 \mathrm{~b} 2$ & $6.5-7$ & 26 & 24 & 684 & 2,559 \\
\hline $452 b 3$ & $11-12$ & 52 & 35 & 1,277 & 4,836 \\
\hline
\end{tabular}


Table 3. Analytical results for partial sequential extractions of Pinal Creek aquifer material--(Continued)

\begin{tabular}{|c|c|c|c|c|c|c|}
\hline Sample & \multicolumn{2}{|c|}{$\begin{array}{l}\text { Depth } \\
\text { (feet) }\end{array}$} & $\begin{array}{l}\mathrm{Al} \text { (carb) } \\
\mathrm{mg} / \mathrm{kg}\end{array}$ & $\begin{array}{l}\mathrm{Al}(\mathrm{MnO}) \\
\mathrm{mg} / \mathrm{kg}\end{array}$ & $\begin{array}{l}\text { Al (amfe) } \\
\mathrm{mg} / \mathrm{kg}\end{array}$ & $\begin{array}{l}\text { Al (cryfe) } \\
\mathrm{mg} / \mathrm{kg}\end{array}$ \\
\hline 452 & \multicolumn{2}{|c|}{$14.8-15$} & 52 & 17 & 1,346 & 8,894 \\
\hline $452 \mathrm{~b} 4$ & 18 & -19 & 31 & 51 & 946 & 4,368 \\
\hline $452 \mathrm{~b} 5$ & 19 & -21 & 35 & 47 & 1,188 & 3,375 \\
\hline $452 \mathrm{~b} 6$ & 23 & -23 & 40 & 25 & 842 & 5,280 \\
\hline $010 \mathrm{bl}$ & 5 & -10 & 37 & 14 & 1,086 & 1,739 \\
\hline $010 \mathrm{~b} 2$ & 15 & -20 & 53 & 22 & 917 & 889 \\
\hline $010 b 3$ & 23 & -25 & 86 & 35 & 1,720 & 7,204 \\
\hline 010b4 & 29 & -30 & 64 & 17 & 1,335 & 1,903 \\
\hline $010 b 5$ & 37 & -38 & 53 & 56 & 1,417 & 7,722 \\
\hline $010 b 6$ & 45 & -46 & 71 & 18 & 1,500 & 6,823 \\
\hline $010 b 7$ & 50 & -52 & 138 & 33 & 1,528 & 4,861 \\
\hline $010 b 8$ & 57. & $5-60$ & 84 & 59 & - & 7,500 \\
\hline 010b9 & 60 & -63 & 35 & 16 & - & 3,940 \\
\hline $010 b 10$ & 63 & -67 & 92 & 28 & - & 8,020 \\
\hline 010b11 & 70 & -75 & 72 & 26 & 581 & 7,558 \\
\hline $010 b 12$ & 86 & -90 & 78 & 11 & - & 8,369 \\
\hline
\end{tabular}

